To be submitted to $A_{1}$.

\title{
Our Sun. IV. The Standard Model and Helioseismology: Consequences of Uncertainties in Input Physics and in Observed Solar Parameters
}

\author{
Arnold I. Boothroyd ${ }^{1}$ and I.-Juliana Sackmann \\ W. K. Kellogg Radiation Laboratory 106-38, California Institute of Technology, \\ Pasadena, CA 91125 \\ aib@krl.caltech.edu \\ ijs@krl.caltech.edu
}

\begin{abstract}
Helioseismic frequency observations provide an extremely accurate window into the solar interior; frequencies from the Michaelson Doppler Imager (MDI) on the Solar and Heliospheric Observatory (SOHO) spacecraft enable the adiabatic sound speed $c$ and adiabatic index $\Gamma_{1}$ to be inferred with an accuracy of a few parts in $10^{4}$, and the density $\rho$ with an accuracy of a few parts in $10^{3}$. This has become a serions challenge to theoretical models of the Sun. Therefore, we have undertaken a self-consistent, systematic study of the sources of uncertainties in the standard solar models. We found that the largest effect on the interior structure arises from the observational uncertainties in the photospheric abundances of the elements (e.g., 15\% uncertainties in C, N, O, and Ne, leading 10 a $\sim 10 \%$ in the $Z / . \mathrm{ratio}$ ), which affect the sound speed profile at the level of 3 parts in $10^{3}$. The estimated $4 \%$ uncertainty in the OPAL opacities could lead to effects of 1 part in $10^{3}$; the $\sim 5 \%$ uncertainty in the basic $p p$ nuclear reaction rate would have a similar effect, as would uncertainties of $\sim 15 \%$ in the diffusion constants for the gravitational settling of helium. The $\sim 50 \%$ uncertainties in diffusion constants for the heavier clements would have nearly as large an effect. Different observational methods for determining the solar radius yield results differing by as much as 7 parts in $10^{4}$;
\end{abstract}

\footnotetext{
'Present address: CITA, U. of Toronto, 60 St. George Street, Toronto, Ontario, Canada M5S 3H8; boothroy@cita.utoronto.ca
} 
wo found that this leads to uncertainties of a few parts in $10^{3}$ in the sound speed in the solar convective envelope, but has negligible effect on the interior. (Wo did not explicitly consider the effects of rotational mixing or uncertainties in the interior equation of state, which other investigators have found to yield uncertainties in the sound speed of order a part in $10^{3}$.) Our reference standard solar model (with $Z / X=0.0245$ ) yielded a convective envelope position $R_{c e}=$ $0.7135 R_{\odot}$, in excellent agreement with the observed value of $0.713 \pm 0.001 R_{\curvearrowright}$, and was significantly affected $\left( \pm 0.003 R_{\odot}\right)$ only by $Z / \mathrm{X}$, the $p p$ rate, and the uncertainties in helium diffusion constants. Our reference model also yielded cnvelope helium abundance $Y_{e}=0.2424$, in good agreement with the range $0.24 \lesssim$ $Y_{c} \lesssim 0.25$ inferred from helioseismic observations; only extreme $Z / X$ values yielded $Y_{c}$ outside this range. We found that other current uncertainties, namely, in the solar age and luminosity, in nuclear rates other than the $p p$ reaction, in the low-temperature molecular opacities, and in the low-density equation of state, have no significant effect on the quantities that can be inferred from helioseismic observations. The predicted pre-main-sequence lithium depletion is uncertain by a factor of 2 . The predicted neutrino capture rate is uncertain by $\sim 30 \%$ for the ${ }^{37} \mathrm{Cl}$ experiment and by $\sim 3 \%$ for the ${ }^{71} \mathrm{Ga}$ experiments (not including uncertainties in the capture cross sections), while the ${ }^{8} \mathrm{~B}$ neutrino flux is uncertain by $\sim 30 \%$.

Subject headings: diffusion - neutrinos - Sun: abundances - Sun: helioseismology -... Sun: interior

\section{Introduction}

Helioseismology provides a powerful tool to explore the deep interior of the Sun. Measurements of solar interior quantities are provided with unprecedented accuracy. The Michelson Doppler Imager (MDI) on the Solar and Heliospheric Observatory (SOHO) spacecraft (Rhodes et al. 1997), the Global Oscillation Network Group (GONG), the Birmingham Solar Oscillation Network (BiSON: Chaplin et al. 1996), and the Low-l instrument (LOWL: Tomczyk et al. 1995) provide helioseismic frequency measurements with accuracies of a few parts in $10^{5}$. From these, the sound speed $c$ throughout most of the solar interior can be inferred with an accuracy of a few parts in $10^{4}$, as can the adiabatic index $\Gamma_{1}$; the density can be inferred with an accuracy of a few parts in $10^{3}$ (Basu, Pinsonneault, \& Bahcall 2000; Bahcall, Pinsonneault, \& Basu 2001).

The ultimate goal of our work was to explore systematically a wide range of solar models 
with relatively modest amounts of mass loss on the early main seduence. and to test their viability via helioseismological measurements (as well ot her observational constraints). This mass loss investigation is presented in our companion paper "Our Sum V' (Sackmann \& Boothroyd 2001). Since the consequences of moderate early solar mass loss are expected to be small, it is essential to understand the consequences of uncertainties in the input physics

and in the input parameters of the solar model. The physics inputs include the equation of state, opacities, nuclear rates, diffusion constants. the treatment of convection (including the possibility of overshoot), and the effects of rotation and mass loss. Observed solar parameters include the solar age and the present solar radius, luminosity, surface composition, and solar wind. A considerable amount of work has been published investigating many of the above effects, as will be discussed in $\S 3$. However, some basic uncertainties still warrant further attention. Before proceeding to our mass loss work, we found it necessary to try to extend the investigations of the above uncertainties; in particular, the major consequences arising from uncertainties in the present observed solar surface $Z / X$ ratio and in the basic $p$ - $p$ chain rate have not been sufficiently considered in the recent (most accurate) work of other investigators. To determine the consequences of these uncertainties. we computed standard solar models with various values of the $\mathrm{Z} / \mathrm{X}$ ratio and the $p$ - $p$ chain rate (lying in the permitted ranges). To obtain a set of self-consistent results, we also investigated the consequences of a number of other uncertainties, namely, in other nuclear rates, in envelope opacities. in the equation of state, in diffusion constants, from different methods of handling interior opacities, and in the solar age. It is the aim of this paper to present the effects of the above uncertainties on the run of the sound speed and density in the solar interior, and on the radius $R_{c e}$ of solar convection and the solar envelope helium abundance $Y_{\text {. }}$. We also present the effects on the solar lithium depletion (excluding rotation effects) and on the production of solar neutrinos. (Note that lithium depletion in a non-rotating standard solar model occurs entirely on the pre-main-sequence; this predicted depletion is significantly smaller than the observed solar lithium depletion factor. Rotational mixing on the main sequence is generally invoked to explain the remaining lithium deficit).

\section{Methods}

We computed a reference standard solar model using up-to-date physics and observed solar parameter values, and several dozen variant solar models in which one of these "inputs" was varied within the allowed errors. (In a few cases, more than one of the "inputs" was varied, or the size of the variation exceeded the size of the quoted errors in order to get a better estimate of the sensitivity.) By comparing with the reference standard solar model, the sensitivity to the uncertainties in the "inputs" could be determined. The stellar evolution 
code used to compute these solar models is descended from that used earlier in our "Sun III" paper (Sackmann, Boothroyd, \& Kraemer 1993; see also Boothroyd \& Sackmann 1999), but has been extensively updated for improved accuracy, including provision for much liner zoning as well as up-to-date input physics.

The reference standard solar model used the OPAL equation of state (Rogers, Swenson, \& Iglesias 1996) in the interior, and the MHD equation of state (Däppen et al. 1988) where it. was designed to be accurate, namely, in the outer envelope at $\log \rho \lesssim-2$ (this corresponds to $r \gtrsim 0.94 R_{\odot}$ and $\log T \lesssim 5.5$ in the present Sun). A version of the MHD equation of state computation program was kindly provided to us (D. Mihalas 1999, private communication), and minor modifications allowed computation of the MHD equation of state for various hydrogen abundances and metallicities down to pre-main-sequence photospheric temperatures and even below (A. I. Boothroyd 2001, in preparation). In both cases, the equation of state was interpolated in metallicity as well as in hydrogen abundance, temperature, and density, in order to take into account metallicity variations due to diffusion and nuclear burning. Variant cases were tested where the OPAL equation of state was used down to $\log T=4$ or even all the way down to its lower limit of validity at $\log T=3.7-$ in the latter case, the MHD equation of state was relevant only to the pre-main-sequence evolution. The MHD equation of state tables computed for this work considered $\mathrm{H}$ and $\mathrm{He}$, plus a 13-element subset of the Grevesse \& Noels (1993) heavy element composition (i.e., C, N, O, $\mathrm{Ne}, \mathrm{Na}, \mathrm{Mg}$, $\mathrm{Al}, \mathrm{Si}, \mathrm{S}, \mathrm{Ar}, \mathrm{K}, \mathrm{Ca}$, and $\mathrm{Fe}$ ); besides the effects due to neutrals and ions, effects due to $\mathrm{H}^{-}$, $\mathrm{H}_{2}$, and $\mathrm{H}_{2}^{+}$were accurately taken into account, and approximate effects of the molecules $\mathrm{C}_{2}, \mathrm{~N}_{2}, \mathrm{O}_{2}, \mathrm{CH}, \mathrm{CN}, \mathrm{CO}, \mathrm{NH}, \mathrm{NO}, \mathrm{OH}, \mathrm{CO}_{2}$, and $\mathrm{H}_{2} \mathrm{O}$ (although these molecules have no effect for solar models: they were actually added in anticipation of use in asymptotic giant branch models). The OPAL equation of state considers only $\mathrm{H}, \mathrm{He}, \mathrm{C}, \mathrm{N}, \mathrm{O}$, and Ne (plus hydrogen-molecule effects); however, the equation of state is quite insensitive to the precise makeup of the metallicity, and the OPAL equation of state may well be the more accurate one in the region where both are valid (Rogers et al. 1996).

The reference standard solar model used the 1995 OPAL opacities "Kopal:GN93" (Iglesias \& Rogers 1996) at temperatures $\log T>4$, and the Alexander \& Ferguson (1994)

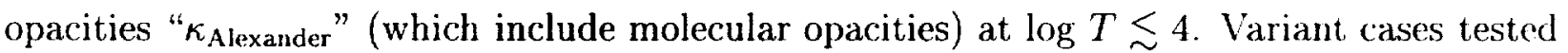
the use of the Sharp (1992) molecular opacities " $\kappa_{\text {Sharp }}$ " instead at $\log T \lesssim 4$, and other high-temperature opacity cases were also considered. The standard 1995 OPAL opacities use the Grevesse \& Noels (1993) heavy element composition for the components of the metallicity $Z$, but the online opacity computation feature of the OPAL web page ${ }^{2}$ allowed

\footnotetext{
${ }^{2}$ http://www-phys.llnl.gov/Research/OPAL/
} 
computation of OPAL opacities appropriate to the more recent Grevesse \& Sauval (1998) mixture ("KOPAL:GS89"); these were tested in variant models, as were opacities appropriate" to the older Grevesse (1984) mixture ("ropaL:Gr84"). The even older and much less accurate

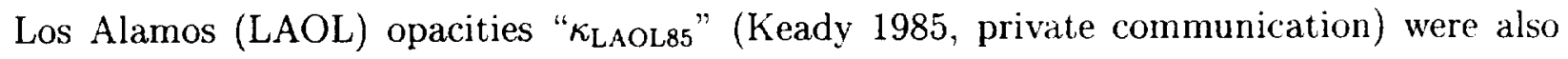
tested in one case.

In our reference standard solar model, we did our best to account for temporal and spatial variations in the opacity due to composition changes from diffusion and nuclear burning. The OPAL opacity tables allow interpolation of the opacity as a function of the hydrogen abundance and the metallicity $Z$; the abundances of the metals comprising $Z$ are always proportional to $Z$ in the OPAL tables, i.e., a "scaled solar" distribution. In addition, the OPAL opacity tables contain mixtures with excess carbon and oxygen (beyond that contained in the scaled solar metallicity), allowing interpolation in carbon and oxygen abundances.

To take into account the variations in abundance due to diffusion. the metallicity value $Z_{\kappa}$ that we used for metallicity interpolation in the OPAL opacity tables was proportional to the abundances of the elements heavier than oxygen: $Z_{\kappa}=Z_{h}$, where $Z_{h} \equiv$ $Z_{0}\left[\sum_{\text {heavy }} X_{i}\right] /\left[\sum_{\text {heavy }}\left(X_{i}\right)_{0}\right]$, where $Z_{0}$ and $\left(X_{i}\right)_{0}$ are the protosolar metallicity and composition, respectively, and $\sum_{\text {heavy }}$ refers to a sum over elements heavier than oxygen. In other words, we scaled the initial solar metallicity by the shift in heavy element abundances resulting from diffusion (note that our diffusion routines assumed that all metals diffused alike). However, for the CNO elements, there are additional changes due to nuclear burning: so that the CNO abundance profiles are not proportional to the heavy element abundance profiles; Turcotte et al. (1998) find that conversion of $\mathrm{C}$ and $\mathrm{O}$ into $\aleph$ results in an opacity change of $\sim 1 \%$ that cannot be modelled by a change in $Z$ alone. These variations in the CNO elements relative to $Z_{h}$ were accounted for in an approximate manner by an additional two-dimensional interpolation in nominal "excess carbon and oxvgen" abundances $\mathrm{C}_{e x}$ and $\mathrm{O}_{e x}$, where these excess abundances account for any variation in the CNO abundancess relative to the scaled solar metallicity $Z_{\kappa}$ of the OPAL opacity tables. Since there were no explicit opacity tables for changes in nitrogen, the best one could do was to distribute excess nitrogen equally between $\mathrm{C}_{e x}$ and $\mathrm{O}_{e x}$ (i.e., to assume that nitrogen opacities were midway between those of carbon and oxygen): $\mathrm{C}_{e x}=\mathrm{C}-\mathrm{C}_{0} \mathrm{Z}_{\kappa} / \mathrm{Z}_{0}+0.5\left(\mathrm{~N}-\mathrm{N}_{0} \mathrm{Z}_{\kappa} / \mathrm{Z}_{0}\right)$ and $\mathrm{O}_{e x}=\mathrm{O}-\mathrm{O}_{0} \mathrm{Z}_{\kappa} / \mathrm{Z}_{0}+0.5\left(\mathrm{~N}-\mathrm{N}_{0} \mathrm{Z}_{\kappa} / \mathrm{Z}_{0}\right)$, such that $\mathrm{CO}_{e x} \equiv \mathrm{C}_{e x}+\mathrm{O}_{e x}=Z-Z_{\kappa}$.

Note that in general, as first carbon and then oxygen is burned to nitrogen, either $\mathrm{C}_{e x}$ or $\mathrm{O}_{e x}$ will be negative, implying extrapolation of the OPAL tables in the direction of zero $\mathrm{C}$ or $\mathrm{O}$ by a non-negligible fraction of $Z$. One might consider the uncertainties in such an extrapolation to be worse than the error inherent in treating all CNO opacities alike; in this 
case, if one of $\mathrm{C}_{e x}$ or $\mathrm{O}_{e x}$ was negative, one would set it to zero and subtract an equivalent amount from the other - this was the method followed in our reference models. However, we also tested the case where negative values were allowed, extrapolating as required. These turned out to yield essentially identical results to the reference model: the rms difference was only 0.00006 in the sound speed profile $\Delta c / c$ and 0.0003 in $\Delta \rho / \rho$, comparable to the estimated numerical accuracy of the models. An alternative case where this latter CNO variation was not approximated by excess $\mathrm{CO}$ at all, i.e., having $Z_{n}=Z_{h}$ but $\mathrm{CO}_{e x}=0.0$. also yielded almost identical results ( $\mathrm{rms}$ difference of $0.00005 \mathrm{in} \Delta c / c$ and 0.0004 in $\Delta \rho / \rho$ ). Similarly negligible rms differences were found for several cases testing different prescriptions for defining $Z_{h}, \mathrm{C}_{e x}$, and $\mathrm{O}_{e x}$ (differing in whether mass fractions or number densities were used, in how the excess nitrogen was divided between $C_{e x}$ and $O_{e x}$, and in whether negative values were allowed for $\mathrm{C}_{e x}$ and $\mathrm{O}_{e x}$ ).

An estimate for the upper limit of the effects of opacity uncertainties was made by simply setting $Z_{\kappa}=f Z$ for a constant factor $f=0.9,0.95,1.0,1.05$, or 1.10 ; note that the case $f=1.0$ is only slightly different from the reference standard solar model.

The reference standard solar model used the NACRE nuclear reaction rates (Angulo et al. 1999), supplemented by the ${ }^{7}$ Be electron capture rates of Gruzinov \& Bahcall (1997) for $\log T \geq 6$ and of Bahcall \& Moeller (1969) for $\log T<6$ (note that this latter lowtemperature region is irrelevant for solar models). Variant models tested cases with nuclear rates changed according to the upper or lower limits quoted in the NACRE compilation; one case tested the use of the older Caughlan \& Fowler (1988) nuclear rates. The program uses the minimum of the weak (Salpeter 1955), intermediate (Graboske et al. 1973), or strong (Itoh et al. 1979; Ichimaru \& Utsumi 1983) screening factors; for solar conditions, this means weak screening, which is a very good approximation to the exact quantum mechanical solution (see, e.g., Bahcall, Chen, \& Kamionkowski 1998b; Gruzinov \& Bahcall 1998). Deuterium was not considered separately (it was assumed to have been entirely burned to ${ }^{3} \mathrm{He}$ on the early pre-main-sequence), but all the other 15 stable isotopes up to and including ${ }^{18} \mathrm{O}$ were considered in detail (i.e., nuclear equilibrium was not assumed for any of them). The other stable isotopes up to ${ }^{28} \mathrm{Si}$ were included in the code (plus a few long-lived unstable isotopes, as well as Fe and a category for the sum of the other elements heavier than Si). but their nuclear reactions were not included since there are no significant effects under solar conditions (except for ${ }^{19} \mathrm{~F}$, which was assumed to be in CNO-cycle nuclear equilibrium for nuclear rate purposes). Neutrino capture cross sections were taken from Bahcall \& Ulrich (1988), except for the ${ }^{8} \mathrm{~B}$-neutrino cross section for capture on ${ }^{37} \mathrm{Cl}$, where the more recent value (5\% higher) of Aufderheide et al. (1994) was used. 
A set of subroutines ${ }^{3}$ were kindly provided to us (M. H. Pinsonneault 1999, private communication) that take into account the diffusion (gravitational settling) of helium and heavy elements relative to hydrogen (see also Thoul, Bahcall, \& Loeb 1994; Bahcall, Pinsonneault, \& Wasserburg 1995). These subroutines assume that all heavy elements diffuse at the same rate as fully-ionized Fe; this yields surprisingly accurate results, as may be seen by comparing to the results of a more detailed treatment (Turcotte \& Christensen-Dalsgaard 1998; Turcotte et al. 1998). The upper limit of the effects of uncertainties in the diffusion constants was estimated by simply multiplying the diffusion constants, either for helium or for the heavy elements, by a constant factor.

The Schwarzschild criterion was used to define convective boundaries; no core overshooting or envelope undershooting was allowed. Note that Morel, Provost, \& Berthomieu (1997) found that including convective core overshooting had a negligible effect on the solar sound speed and density profiles, but on the other hand that including convective envelope undershooting by even a tenth of a pressure scale height moved the solar convective envelope boundary inwards by eight times the uncertainty in the observed value vielding a sharp spike in the difference between observed and calculated sound speed profiles. Rotation-induced mixing was not considered in our models; the effect that it would have is discussed in $\S 3.1$.

The reference standard solar model used a value of $Z / X=0.0245$ for the the present solar surface metals-to-hydrogen ratio (by mass fraction), as given by Grevesse \& Noels (1993). Variant models tested a $10 \%$ lower value of $Z / X=0.022$, and the $\sim 13 \%$ higher older value of $Z / X=0.0277$ (Grevesse 1984; Anders \& Grevesse 1989). Additionally, variant models with the value $Z / X=0.023$ recommended by Grevesse \& Sauval (1998) were considered. The presolar abundances of the heavy elements were taken to be the ones used in the OPAL opacities used in the solar model; for the reference standard solar model and most variants, this was the mix of Grevesse \& Noels (1993). A variant model with $Z / X=0.023$ also tested use of the heavy element mix and OPAL opacities appropriate to the abundances reported by Grevesse \& Sauval (1998), and a variant model with $Z / X=0.0277$ tested use of the heavy element mix and OPAL opacities appropriate to the abundances reported by Grevesse (1984). Tests were made with C, N, O, and Ne abundances increased or decreased by their uncertainties of $15 \%$ relative to $\mathrm{Fe}$ (with the OPAL opacities appropriate to these revised mixes).

A present solar mass of $M_{\odot}=1.9891 \times 10^{33} \mathrm{~g}$ (Cohen \&. Taylor 1986) was used in all cases. The uncertainty of $0.02 \%$ is too small to have any significant effect, and is in fact smaller than the amount of mass lost by the sun since it was formed in the form of radiation

\footnotetext{
${ }^{3}$ These subroutines are available from Bahcall's web page: http://www.sns.ias.edu/ $\sim$ jnb/
} 
alone (photons and neutrinos: $\Delta M=\Delta E / c^{2}$ ). Mass loss from this effect and from the solar wind was ignored for all cases considered in this paper; as is shown in our companion paper "Our Sun V" (Sackmann \& Boothroyd 2001), it would have negligible effect, even if the average solar wind over the past $4.6 \mathrm{Gyr}$ had been an order of magnitude higher than its present value, as is suggested by measurements of noble gas isotopes in lunar rocks (Geiss 1973; Geiss \& Bochsler 1991; Kerridge et al. 1991).

For the reference standard solar model and most variant cases, a present solar luminosity of $L_{\odot}=3.854 \times 10^{33} \mathrm{erg} \mathrm{s}^{-1}$ was used, as discussed in Sackmamn et al. (1993). A value $0.3 \%$ lower $\left(3.842 \times 10^{33} \mathrm{erg} \mathrm{s}^{-1}\right)$ with an estimated $1-\sigma$ uncertainty of $0.4 \%$ was recently obtained by Bahcall et al. (2001), based on the observations of Fröhlich \& Lean (1998) and Crommelynck et al. (1996). Variant models considered the effect of using this more recent solar luminosity value, and high and low values $2-\sigma(0.8 \%)$ above and below it. Note that most of the uncertainty in $L_{\odot}$ comes not from uncertainties in the present solar irradiance, but rather from uncertainties in the slight long-term variability of the solar luminosity. For example, Lean (2000) estimates a difference of $0.2 \%$ between the present value and that during the seventeenth century Maunder Minimum, and even this timescale is much shorter than the Sun's Kelvin-Helmholtz timescale of $\sim 10 \mathrm{Myr}$.

The reference standard solar model used a solar radius at the photosphere $(\tau=2 / 3)$ of $R_{\odot}=695.98 \mathrm{Mm}$ (Ulrich \& Rhodes 1983; Guenther et al. 1992). Variant models considered the effect of using the value of $695.78 \mathrm{Mm}$ suggested by the helioseismic $f$-mode study of Antia (1998), or the value of $695.508 \mathrm{Mm}$ suggested by the solar-meridian transit study of Brown \& Christensen-Dalsgaard (1998).

In his Appendix to Bahcall et al. (1995), G. J. Wasserburg provides a systematic analysis of the upper and lower bounds on the age of the Sun, as obtained from isotopic ratios measured in meteorites. We paraphrase his discussion in this paragraph: The protosolar nebula (out of which the Sun and the meteorites formed) contained not only the stable isotope ${ }^{27} \mathrm{Al}$ but also the unstable isotope ${ }^{26} \mathrm{Al}$, which decays into ${ }^{26} \mathrm{Mg}$ with a half-life of only $0.7 \mathrm{Myr}$. This ${ }^{26} \mathrm{Al}$ must have been injected into the protosolar nebula from the stellar source where it was created. Isotopic measurements of meteoritic crystallized refractory condensates show that they had a ratio ${ }^{26} \mathrm{Al} /{ }^{27} \mathrm{Al}=5 \times 10^{-5}$ at the time they formed. Even if the stellar source of the ${ }^{26} \mathrm{Al}$ had a very high ratio ${ }^{26} \mathrm{Al} /{ }^{27} \mathrm{Al} \sim 1$, the time interval between the formation of the ${ }^{26} \mathrm{Al}$ and the formation of the meteorite cannot have exceeded $\sim 11 \mathrm{Myr}$. The Sun cannot have formed later than these meteorites. However, the Sun/meteorite system must have formed after the injection of ${ }^{26} \mathrm{Al}$ into the protosolar nebula, so the Sun cannot have formed earlier than $\sim 11$ Myr before the formation of these meteorites. The age of the meteorites has been accurately measured using ${ }^{207} \mathrm{~Pb} /{ }^{206} \mathrm{~Pb}$ ratios, to be $4.565 \pm 0.005 \mathrm{Gyr}$. 
It follows that the Sun cannot have formed more than 4.59 Gyr ago, nor later than 4.55 Grir ago (i.e., $4.565+3 \times 0.005+0.011$ Gyr. or $4.565-3 \times 0.005$ Gyr).

Our solar models were started relatively high on the pre-main-sequence Havashi track: with central temperatures below $10^{6} \mathrm{~K}$; note that the ages $t_{\odot}$ of all our models are quoted relative to this pre-main-sequence starting point, which is within a few Myr of the solar formation age constrained by the meteoritic ages discussed above. A relatively high value of $t_{\odot}=4.6 \mathrm{Gyr}$ was used for the reference standard solar model; to get a reliable estimate of the sensitivity of the models to the solar age uncertainty, variant models were computed with ages differing by very large amounts, namely, $t_{\odot}=4.5$ and $4.7 \mathrm{Gyr}$.

From our Hayashi track starting point, it takes only $\sim 3$ Myr for the luminosity on the pre-main-sequence to drop below $1 L_{\odot}$ (i.e., below the present solar luminosity), but much longer, namely an additional $40 \mathrm{Myr}$, to reach the zero-age main sequence (ZAMS) -- we have defined the ZAMS as the stage where the pre-main-sequence contraction terminates and the Sun begins to expand slowly, as nuclear burning in the core (rather than gravitational (ontraction) supplies essentially all of the solar luminosity. For the next $\sim 50 \mathrm{Myr}$, (rolution is fairly fast, as $p+\mathrm{C}$ reactions burn the initial carbon to nitrogen near the Sun's center, resulting in a short-lived convective core. Subsequently, the carbon abundance approaches its CN-cycle equilibrium abundance, the convective core disappears, and the Sun settles down to burn hydrogen mainly via the $p p$-chain reactions. Note that the pre-main-sequence timescale implies that the total solar age $t_{\odot}$ used in this paper can be converted into a main sequence solar lifetime by subtracting about 0.04 Gyr.

We compared our solar models to profiles of the solar sound speed $c_{6}$, density $\rho_{(-)}$, and adiabatic index $\left(\Gamma_{1}\right)_{\odot}$ obtained from the helioseismic reference model of Basu et al. $(2000)^{4}$. which they obtained by inversion from the helioseismic frequency observations. In the inversion process, a standard solar model is required, but Basu et al. (2000) demonstrated that the resulting $c_{\odot}$ and $\rho_{\odot}$ profiles of the helioseismic reference model are relatively insensitive to uncertainties in the standard solar model used for this purpose (except for uncertainties in $R_{\odot}$, as discussed in $\S 3.1$ ). They estimated a net uncertainty of few parts in $10^{4}$ for the sound speed $c_{\odot}$ and adiabatic index $\left(\Gamma_{1}\right)_{\odot}$, and a few parts in $10^{3}$ for the density $\rho_{\odot}$. However, in the Sun's core $\left(r \lesssim 0.1 R_{\odot}\right)$, systematic uncertainties in the helioseismic sound profile are increased by a factor of $\sim 5$; this was demonstrated by Bahcall et al. (2001), who compared helioseismic inversions of different helioseismic data sets. We used their comparison to estimate the $r$-dependence of the systematic error in $c_{\odot}$ in the core and in the convective envelope (namely, a fractional systematic error decreasing linearly from 0.0013 at

\footnotetext{
${ }^{4}$ From the denser-grid machine-readable form of their Table 2, at http://www.sns.ias.edu/ jnb/
} 
$r=0.05 R_{\odot}$ to 0.0003 at $r=0.2 R_{\odot}$, constant from there to $r=0.72 ; R_{\odot}$, then increasing linearly to 0.00052 at $\left.r=0.94 ; R_{\odot}\right)$. For $c_{\odot}$, this systematic error can be significantly larger than the statistical errors, and we combined the two in quadrature to get the fractional error $\left(\sigma_{c} / c\right)$ for the purpose of calculating weighted rms differences -- the rms fractional difference in $c$ is given by $\left(\left\{\sum\left[(\Delta c / c) /\left(\sigma_{c} / c\right)\right]^{2}\right\} /\left\{\sum\left[1 /\left(\sigma_{c} / c\right)\right]^{2}\right\}\right)^{1 / 2}$. For $\left(\Gamma_{1}\right)_{\odot}$ and $\rho_{\odot}$, the systematic errors are comparable to or smaller than the statistical ones, and the statistical errors sufficed for calculating weighted rms differences.

\section{Results and Discussion}

\subsection{Sound Speed and Density Profiles}

We present in Figures 1 through 5 profiles of the adiabatic sound speed differences $\delta c / c \equiv\left(c_{\odot}-c_{\text {model }}\right) / c_{\odot} ;$ profiles of the density differences $\delta \rho / \rho \equiv\left(\rho_{\odot}-\rho_{\text {model }}\right) / \rho_{(\odot)}$ are available online ${ }^{5}$. For our equation of state comparison, we also considered the equivalent fractional difference in the adiabatic index $\Gamma_{1}$. The theoretical sound speeds $c_{\text {model }}$ and densities $\rho_{\text {model }}$ are from our computed reference standard solar model and from our variant standard solar models. Our reference standard solar model used current input parameters, as discussed in $\S 2$; our variant standard solar models comprised standard solar models with one or more input parameters varied within the permitted range. We present all our sound speed and density profiles in terms of differences relative to the observed helioseismic reference profiles. This choice of presentation not only allows one to see the effects of the uncertainties in the input parameters, but also shows which choice of input parameters agrees best with the helioseismic observations.

Rotation effects: The prominent peak in $\delta c / c$ at $r \sim 0.7 R_{\odot}$ visible in Figure la is due to the neglect of rotation-induced mixing just below the base of the solar convective envelope, as has been shown by a number of investigators who have included parameterized rotational mixing (see, e.g., Richard et al. 1996; Brun, Turck-Chièze, \& Zahn 1999; Basu et al. 2000; Bahcall et al. 2001). There are significant uncertainties in the physical processes that lead to rotation-induced mixing. However, all of these investigators agree that rotational mixing is capable of smoothing out the peak at $r \sim 0.7 R_{\odot}$, and that this has a relatively small effect on the sound speed elsewhere in the Sun (a fractional change of less than 0.001). For example, Bahcall et al. (2001) found that including "maximal" rotational mixing spread

\footnotetext{
${ }^{5}$ http://www.krl.caltech.edu/ aib/papdat.html
} 
out this peak over the region $0.3 R_{\odot} \lesssim r \lesssim 0.7 R_{\odot}$. eliminating the prominent peak but worsening the agreement with the helioseismological sound speed profile by about 0.001 in much of the solar interior $\left(0.3 R_{\odot} \lesssim r \lesssim 0.6 R_{\odot}\right)$. The "minimal" mixing model of Richard et al. (1996) yielded much the same result, as did a similar model of Brun et al. (1999). As far as the core and the convective envelope are concerned, rotational mixing should have no significantly effect, as shown by the above authors.

Since the prominent peak at $r \sim 0.7 R_{\odot}$ results from the neglect of rotational mixing, we did not require agreement in this region between profiles from our theoretical models and profiles inferred from the helioseismic observations. Nor did we require agreement in core region, since the present helioseismic observations still result in large uncertainties in the inferred profiles there; for example, as shown by Bahcall et al. (2001), the use of a different helioseismic dataset could remove the the sharp upturn in $\delta c / c$ for $r \lesssim 0.1 R_{\odot}$ in Figure la (and even convert it into a downward trend). On the other hand, we aimed for agreement in the regions $0.1 R_{\odot} \lesssim r \lesssim 0.6 R_{\odot}$ and $0.72 R_{\odot} \lesssim r \lesssim 0.94 R_{\odot}$, where disagreements are due to imperfections in the input physics or uncertainties in the observed solar parameters. This is demonstrated by our variant models and by the variant models of Morel et al. (1997) and Basu et al. (2000).

Convergence accuracy effects: The accuracy with which the model is converged to the solar radius, luminosity, and $Z / X$ values can affect the sound speed profiles. The extent to which this occurs depends on the accuracy of the convergence, and on the sensitivity of the profiles to variations in $R_{\odot}, L_{\odot}$, and $Z / X$ (these are discussed in detail below). Our convergence accuracy resulted in effects no larger than a few parts in $10^{5}$ on the sound speed in the solar interior $\left(r \lesssim 0.6 R_{\odot}\right)$. In the convective envelope, where the sound speed is quite sensitive to $R_{\odot}$, the effect was typically less than a part in $10^{4}$ (this included the reference standard solar model), but could be as high as a few parts in $10^{4}$ for the few models with the worst convergence in $R_{\odot}$. This was determined not only by the sensitivity considerations discussed below, but also by considering models with differing degrees of convergence on $R_{\odot}$, $L_{\odot}$, and $Z / X$.

Note that the sound speed profile in most of the solar convective envelope $\left(0.72 R_{6} \lesssim\right.$ $\left.r \lesssim 0.94 R_{\odot}\right)$ is sensitive mainly to the equation of state and to the solar radius, with other uncertainties having only a minor effect there, as can be seen by considering this subregion in Figures 1 through 5.

Zoning effects: We investigated the effects of using two different zonings. Our coarsezoned models had about 2000 spatial zones in the model, and about 200 time steps in the 
evolution from the zero-age main sequence to the present solar age (plus about 800 time steps on the pre-main-sequence). Typically, these models were converged to match the solar luminosity and radius to about a part in $10^{5}$, and the solar surface $Z / X$ to a part in $10^{4}$; a few cases where convergence was slow were nearly 10 times worse. Our fine-zoned models had 10000 spatial zones and took 1500 main-sequence time steps (plus 6000 pre-main-sequence time steps) - a factor of 5 increase in both spatial and temporal precision -... and were typically converged to better than a part in $10^{5}$ for $R_{\odot}$ and $L_{\bullet}$, and a few parts in $10^{5}$ for $Z / X$. We also tested some very coarse-zoned models, with 1000 spatial zones, 100 mainsequence time steps (plus 600 pre-main-sequence time steps), and convergence to the solar parameters of a few parts in $10^{4}$. (A coarse-zoned converged solar model took a few hours of CPU-time on a high-performance ES40 computer, as compared to about four days of CPU-time for a fine-zoned converged model; these times were roughly tripled on a $450 \mathrm{Mhz}$ Pentium III PC.)

Figure 1a shows that the fine zoning made only a very modest improvement relative to the coarse-zoned case, less even than the statistical errors in the sound speed and density profiles obtained from helioseismic inversions. Even the very coarse-zoned test case did not do too badly: in the solar interior, it differs from the fine-zoned case by no more than 0.0004 in the sound speed profile and 0.003 in the density profile (amounts comparable to the systematic uncertainties in the helioseismic inversion) - the rms differences (over the entire Sun) are even smaller, at 0.0003 and 0.002 , respectively. The coarse-zoned model did about 3 times better still, with rms differences relative to the fine-zoned case of 0.0001 and 0.0008 for $\delta c / c$ and $\delta \rho / \rho$, respectively. Zoning changes had no effect on the adiabatic index $\Gamma_{1}$ (the coarse-zoned $\Gamma_{1}$ curves were not plotted in Fig. 1b, since they would be precisely superimposed on the fine-zoned curves). Additional tests demonstrated that different zoning always led to the same negligibly small systematic shift in solar interior $\delta c / c$ and $\delta \rho / \rho$ values (although inaccuracies in matching the observed solar surface parameters could lead to slightly larger random variations in the convective envelope region $r \gtrsim 0.7 R_{\odot}$ ). We therefore felt justified in running most of the models with our coarse zoning. Note that Morel et al. (1997), with about 1000 spatial zones, 60 main-sequence time steps, and convergence to present solar surface parameters of a part in $10^{4}$ (similar to our very-coarse-zoned case), claimed a numerical internal accuracy of 0.0005 in the sound speed, similar to what we found.

Equation-of-state effects: Morel et al. (1997) compared two relatively-current equations of state, and found a small but non-negligible effect. They compared the OPAL equation of state (Rogers et al. 1996) with the CEFF equation of state (Christensen-Dalsgaard \& Däppen 1992), finding differences of slightly over 0.001 in the sound speed profile and up to 0.01 in the density. (Note that they had set the value of $Z_{\text {eos }}$ used in their equation 
of state to a fixed value of 0.019 ; however, since the equation of state is only very weakly sensitive to $Z$, this should have only a minor effect on their models, and should not affect their comparison of the two equations of state.)

Guzik \& Swenson (1997) presented more extensive comparisons, relative to the OPAL equation of state, of both their own SIREFF equation of state and the MHD equation of state of Däppen et al. (1988) (they too used a fixed $Z_{\text {eos }}$, of 0.02 , but again this should not affect the comparison). They likewise found an effect $\Delta c / c \leq 0.001$ at $r \lesssim 0.95 R$. (with differences up to 0.004 near the surface, probably due to the different methods used to determine ionization effects, which are very large there due to the helium and hydrogen ionization zones). They also presented differences between the values of the pressure $P$; specific heat at constant pressure $C_{p}$, internal energy $U$, and adiabatic index $\Gamma_{1}$. As one might expect from the sound speed, differences between equations of state near the solar surface $\left(r>0.95 R_{\odot}\right)$ were relatively large, of order $1 \%$. However, for $r \lesssim 0.95 R_{\odot}$ Guzik \& Swenson (1997) reported OPAL - MHD differences $\Delta P / P \leq 0.0015, \Delta U / U \leq 0.002$, $\Delta \Gamma_{1} / \Gamma_{1} \leq 0.0007$, and $\Delta C_{p} / C_{p} \leq 0.015$ (with $\Delta C_{p} / C_{p} \leq 0.006$ for $r \lesssim 0.9 R_{\text {.. }}$ ): the OPAL - SIREFF differences were slightly smaller for $C_{p}$, slightly larger for $P$ and $U$, and much larger (a factor of $\sim 3$ ) for $\Gamma_{1}$. For $r \gtrsim 0.3 R_{\odot}$, the SIREFF value of $\Gamma_{1}$ has several relatively large "wiggles" (fractional variations $\sim \pm 0.002$ ) relative to either OPAL or MHD; but most of the difference in the core, and perhaps some difference in the average trend further out, may be due to the fact that SIREFF includes relativistic electron effects, while MHD and OPAL do not. Elliot \& Kosovichev (1998) estimated that inclusion of relativistic effects would reduce the MHD or OPAL value of $\Gamma_{1}$ by a fraction 0.002 at $r \approx 0.1 R_{\sigma}$, this correction growing less with increasing $r$, to reach 0.001 at $r \approx 0.3 R_{\odot}$ and zero near the solar surface. They pointed out that such a shift in $\Gamma_{1}$ for models using the OPAL or MHD equation of state would significantly improve the agreement in the solar interior with the inferred helioseismic $\Gamma_{1}$ profile. Certainly, if such a correction were applied to our $\Gamma_{1}$ curve in Figure 1b, the model profile would agree with the helioseismic: profile within the statistical errors for all $r \lesssim 0.6 R_{\odot}$ (recall that decreasing a model quantity shifts the curve upwards in the figure).

The adiabatic sound speed is defined as $c=\left(\Gamma_{1} P / \rho\right)^{1 / 2}$; changes in the solar ratio of $P / \rho$ would result not only from changes in the equation of state but also from readjustments of the solar structure in response to these changes, so it is not obvious a priori what effect relativistic corrections would have on the sound speed. Consideration of the effect from $\Gamma_{1}$ alone suggest that relativistic corrections might reduce the slope at $r \lesssim 0.5 R_{\odot}$ in the $\delta c / c$ curve of Figure 1a. The sound speed differences presented by Guzik \& Swenson (1997) for their OPAL - SIREFF comparison suggest that this would in fact be the case, and that a fractional decrease of order 0.001 in the sound speed $c_{\text {model }}$ near the Sun's center (i.e., 
an increase of $0.001 \mathrm{in} \delta c / c$ there) would result from relativistic corrections to the OPAL equation of state.

Richard et al. (1998) compared the OPAL value of $\Gamma_{1}$ with the MHD value in the convective envelope $\left(0.72 R_{\odot} \lesssim r \lesssim 0.98 R_{\odot}\right)$ resulting from the equation of state with that from the MHD equation of state (Däppen et al. 1988), finding that the OPAL equation of state appeared to perform slightly better. This is not very surprising; what is perhaps more surprising is how well the MHD equation of state does in the solar interior, since it was not originally designed to be accurate for $\rho \gtrsim 10^{-2} \mathrm{~g} \mathrm{~cm}^{-3}$ (i.e., $r \lesssim 0.94 R_{\odot}$ and $\log T \lesssim 5.5$ in the Sun). We investigated the effect of changing the equation of state only in this outer region where both are expected to be valid.

Our reference standard solar model used the OPAL equation of state in the interior regions, switching over to the MHD equation of state in the outer envelope; this switchover was performed gradually over the region $-1.5>\log \rho>-2$ (corresponding to $5.8>\log T>$ 5.5 in the present Sun). We compared this reference standard model with two cases where the switchover occurred even further out in the envelope: a case "OPALcos-midT" where the switchover occurred for $4.0>\log T>3.9$, and a case "OPALeos-low T" where the switchover occurred for $3.75>\log T>3.7$. In this latter "OPALeos-lowT" case, the MHD equation of state is used only outside the Sun's photosphere, and thus has negligible effect on the main sequence evolution. On the other hand, any artifacts induced by the switchover might be smaller in the former "OPALeos-midT" case, since differences between the two equations of state are significantly less near $\log T=4$ than near $\log T=3.7$. Note that, while the OPAL opacity tables are unreliable for $\log T \lesssim 4$ due to their neglect of molecular opacities, the OPAL equation of state tables include molecular hydrogen effects, and should be accurate down to their lower tabulation limit of $\log T=3.699$ (Rogers et al. 1996).

Figure 1 shows that, as one would expect, changing the equation of state in the outer envelope alone has no effect on the interior, and only a minor effect in that part of the convective envelope where the equation of state remains unchanged. (Since the effects were so small, we computed fine-zoned cases for this equation-of-state test; these are the ones presented in Fig. 1.) The OPAL equation of state appears to yield slightly better $\Gamma_{1}$ values than the MHD equation of state in the envelope at $r>0.9 R_{\odot}(\log \rho<-1.5)$. We agree with Guzik \& Swenson (1997) in finding maximum differences between these two equations of state in the outer solar envelope (at $\log T \sim 5)$ of about $1 \%$ in pressure $P$ and in $\chi_{\rho} \equiv(\partial \ln P / \partial \ln \rho)_{T}$, and of $2 \%$ in the specific heat at constant volume $C_{v}$ and in $\chi_{T} \equiv(\partial \ln P / \partial \ln T)_{\rho}$.

Solar radius effects: Basu et al. (2000) demonstrated that using a solar radius different from the standard value of $R_{\odot}=695.98 \mathrm{Mm}$ could have a small but not completely 
insignificant effect on both the sound speed profile inferred from helioseismic inversions and that computed in solar models. They found that using the $0.03 \%$ smaller solar radius value $R_{\odot}=695.78 \mathrm{Mm}$ (case "R78") suggested by the $f$-mode study of Antia (1998) would reduce the inferred helioseismic sound speed profile throughout the Sun by about the same small fraction, namely 0.0003 (nearly independent of position in the Sun); similarly, using the $0.07 \%$ smaller value $R_{\odot}=695.508 \mathrm{Mm}$ (case "R508") suggested by the solar-meridian transit study of Brown \& Christensen-Dalsgaard (1998) would reduce the inferred helioseismic sound speed profile by 0.0007 . On plots such as ours of fractional differences $\delta c / c \equiv\left(c_{6}-c_{m o d e l}\right) / c_{(\sigma)}$, such a reduction in " $c_{\odot}$ " would shift all the curves downwards by the given amounts. This shift has not been performed in our diagrams - we only display our c model values relative to the $c_{\odot}$ values of Basu et al. (2000) - but this effect has been included in our quoted rms values relative to the Sun. Basu et al. (2000) also calculated that a change in the solar radius would result in a non-uniform shift in the inferred helioseismic $\rho_{\odot}$ and $\left(\Gamma_{1}\right)_{\odot}$ profiles, by amounts comparable to the statistical errors in these quantities; although these shifts are barely significant statistically, in contrast to the shift in $c_{\odot}$, for completeness their effects have been applied to our rms values calculated rolative to the helioscismic profiles for the "R78" and "R508" cases.

Our "R78" and "R508" models show that changing the value of $R_{\odot}$ has no effect on $\Gamma_{1}$, and only in the convective envelope does it affect $c$ and $\rho$ significantly, with the largest effect being near the solar surface. The effect on $c$ is shown in Figure 1a. For clarity, these "R78" and "R508" curves are omitted at $r<0.72 R_{\odot}$; in the "peak" region, they differ from the reference standard solar model by a few parts in $10^{4}$, and this difference drops to about a part in $10^{5}$ for $r<0.6 R_{\odot}$. In the convective envelope, the "R78" case is an improvement on the reference model (reducing the rms error in $\delta c / c$ from 0.0007 to 0.0004 when one includes the effect of the shift in the inferred helioseismic profiles), but the "R508" case is worse ( $\mathrm{rms}$ of 0.0011). For the "OPALeos-midT" case, reducing the solar radius always worsens agreement in the convective envelope (an envelope rms of 0.0003 is increased to 0.0009 or 0.0018 for "R78" or "R508," respectively). The overall rms and the rms in the interior are not much affected, as may be seen from Table 1; however, if relativistic corrections had been included in the equation of state, a decrease in the solar radius probably would have led to some improvement.

Solar luminosity effects: Bahcall et al. (2001) tested the effect of $2-\sigma$ changes in the value of $L_{\odot}$ (namely, $\pm 0.8 \%$ ) on their solar models, finding only a minor effect on neutrino fluxes and negligible effects on the other quantities they considered. For completeness, we made the same test with our own models, confirming their results. Figure 1a shows the effects on the sound speed of a solar luminosity $0.8 \%$ lower (" $L_{\text {low }}$ ") and $0.8 \%$ higher (" $L_{h i g h}$ ") than 
the most recent value of $3.842 \times 10^{33} \mathrm{erg} \mathrm{s}^{-1}$ (Bahcall et al. 2001; Fröhlich \& Lean 1998; Crommelynck et al. 1996) - note that our reference standard solar model lies closer to the latter than to the former, as it uses a slightly higher $L_{\odot}$ value than the most recent estimate (see $\S 2)$. To avoid confusion with other curves, the " $L_{\text {high" }}$ and " $L_{l o w}$ " curves are shown only in the region where they differ the most, namely, $r \lesssim 0.3 R_{\odot}$; even in this region, a shift of $0.8 \%$ in $L_{\odot}$ produces a fractional change in the sound speed of less than 3 parts in $10^{4}$, dropping to 1 part in $10^{4}$ for $r>0.3 R_{\odot}$.

Solar age effects: Figure 1a demonstrates that the uncertainty in the solar age $t_{\odot}$ has only a very minor effect on the solar sound speed profile - note that the shift illustrated here, from $t_{\odot}=4.6 \mathrm{Gyr}$ to $4.5 \mathrm{Gyr}$, is much larger than the observationally allowed range of solar ages (i.e., $4.55 \mathrm{Gyr}<t_{\odot}<4.59 \mathrm{Gyr}$ ) discussed in $\S 2$. (These ages are defined to include the pre-main-sequence; main sequence ages can be obtained by subtracting $0.04 \mathrm{Gyr}$ ). The maximum allowed shift of $0.02 \mathrm{Gyr}$ relative to the "best" solar age of $4.57 \mathrm{Gyr}$ would yield negligibly small effects, namely, an rms of 0.0001 for $\Delta c / c$ and an $\mathrm{rms}$ of 0.001 for $\Delta \rho / \rho$. with maximum changes less than twice these values. Our results agree both qualitatively and quantitatively with the age sensitivity found in the recent work of Morel et al. (1997).

Low-temperature opacity effects: Uncertainties in the low-temperature molecular opacities appear to have negligible impact on the structure of a solar model. Using the Sharp (1992) molecular opacities (" $\kappa$ Sharp") below $10^{4} \mathrm{~K}$ rather than the Alexander $\&$ Ferguson (1994) molecular opacities (" $\kappa_{\text {Alexander }}$ ") led to essentially identical sound speed and density profiles -- the " $\kappa_{\text {Sharp }}$ " case is thus not plotted in Figure 2 . The rms differences are negligible, less than a part in $10^{4}$ for the sound speed and less than a part in $10^{3}$ for the density.

Interior opacity effects: Recently, Morel et al. (1997) have demonstrated the serious impact of opacity changes on the sound speed and density profiles of solar models. They compared the 1995 OPAL opacities (Iglesias \& Rogers 1996; Rogers et al. 1996) with the less-precise 1992 OPAL opacities (Rogers \& Iglesias 1992) (albeit with models that neglected diffusion), finding that the improved opacities made an improvement of up to 0.005 in the sound speed and up to 0.03 in the density (see their models S1 and S2). Basu et al. (2000) compared a model with the 1995 OPAL opacities and the OPAL equation of state (Rogers et al. 1996) to a model with the 1992 OPAL opacities and the cruder Yale equation of state (Guenther et al. 1992) with the Debye-Hückel correction (Bahcall, Bahcall, \& Shaviv 1968) (their models did include diffusion); they likewise found an effect of up to 0.005 in the sound speed and up to 0.03 in the density, from the combination of these two changes 
in the input. We found that an even larger improvement of up 100.007 in the somd speed resulted from changing from the even older Los Alamos (LAOL) opacities (Keady 1985, private communication) to the 1995 OPAL opacities. with an rms improvement of 0.004 as shown in Table 1. (there is also an improvement of up to 0.04 in the density, with an rms improvement of 0.02). However, such a large opacity change overestimates the uncertainty in the 1995 OPAL opacities (Rogers \& Iglesias 1998).

As pointed out by Morel et al. (1997), neglecting the opacity changes that result from metallicity variations in the Sun would lead to significant errors ...- e.g., errors of up to 0.0015 in the sound speed. In our reference standard solar model, we did our best to account for these temporal and spatial variations in the opacity due to these composition changes from diffusion and nuclear burning. The metallicity value $Z_{\kappa}$ that we used for metallicity interpolation in the OPAL opacity tables was scaled according to the changes in the elements heavier than oxygen (resulting from diffusion): $Z_{\kappa}=Z_{h}$, where $Z_{h} \equiv Z_{0}\left[\sum_{\text {heavy }} X_{i}\right] /\left[\sum_{\text {heavy }}\left(X_{i}\right)_{0}\right]$. The extent to which the CNO element abundances differ from the scaled solar values in $Z_{h}$ was accounted for by an extra interpolation in nominal "excess carbon and oxygen" abundances $\mathrm{C}_{e x}$ and $\mathrm{O}_{e x}$ using the OPAL opacity tables for extra $\mathrm{C}$ and/or $\mathrm{O}$ abundances. (Several similar methods of doing this were considered; as discussed in $\S 2$, all gave essentially identical results - even omitting the CNO-correction entirely had almost no effect.)

An alternative approximation ("approx- $\kappa$ ") is to set $Z_{\kappa}=Z$ (i.e., to interpolate the OPAL opacity tables in the local metallicity $Z$, but ignore effects of variations in the makeup of $Z$ ). As shown in Figure 2, this approximation yields results almost identical to those of our reference standard solar model; the rms difference was only $0.0002 \mathrm{in} \Delta c / c$ and $0.001 \mathrm{in}$ $\Delta \rho / \rho$. Morel et al. (1997) compared two different ways of estimating the value of $Z_{\kappa}$ (both being similar but not identical to our "approx- $\kappa$ " case); they likewise found only very minor differences in the sound speed between their two methods, but much larger effects on the density (see their models D3 and D12).

Even when using the most up-to-date OPAL opacities, one can still get significant errors if one neglects the effect on opacity of $Z$-changes (primarily due to diffusion). The simplest case is to set $Z_{\kappa}=Z_{0}$ ("const- $Z_{\kappa}$ "); in effect, such a case uses only the OPAL opacity tables relevant to the protosolar metallicity and ignores the effect on the opacity of any subsequent changes in the metallicity. Figure 2 demonstrates that this "const- $Z_{\kappa}$ " case yields errors of up to $\sim 0.0015$ in $\Delta c / c$ relative to the more accurate opacity interpolation of the reference standard solar model, in agreement with the results of Morel et al. (1997) (compare their models D10 and D12); the rms differences were 0.0010 in $\Delta c / c$ and 0.007 in $\Delta \rho / \rho$. One might attempt to fix up this neglect of metallicity variation by interpolation using the mildly $\mathrm{CO}-$ enhanced OPAL opacity tables, i.e., retaining a constant $Z_{\kappa}=Z_{0}$ for opacity interpolation 
purposes but setting $\mathrm{CO}_{e x}$ to the difference between $Z_{\kappa}$ and the true value of $Z$ that results from diffusion and nuclear burning, i.e., $\mathrm{CO}_{e r}=Z-Z_{\kappa}=Z-Z_{0}$. Figure 2 illustrates that this approximation is a slight improvement over the "const- $Z_{\kappa}$ " case but still not very satisfactory: rms differences relative to the reference standard solar model are 0.0006 in $\Delta c / c$ and 0.004 in $\Delta \rho / \rho$.

Interpolation errors can also arise from the finite grid spacing of the opacity tables in $X, Z, T$, and $\rho$. In creating the opacity interpolation routines, we tested the $X$ - and $Z$ interpolation, finding that these should result in only minor errors (a fraction of a percent).

In addition to errors introduced by methods of interpolating in opacity tables, one must consider the crrors in the actual opacity values contained in the tables. Such opacity errors will in general be functions of temperature and density; also, different elements will have different errors. Rogers \& Iglesias (1998) estimate that there is a 4\% uncertainty in the 1995 OPAL opacities from effects neglected in their calculations. Turcotte et al. (1998) showed that differences in the Rosseland mean opacities between 1992 and 1995 OPAL opacities do not exceed $7 \%$ over the run of temperature and density in the Sun's interior, and that these opacity differences yielded sound speed differences of up to 0.002 in their solar models. In addition, errors in the observed relative heavy element abundances in the solar envelope will translate into opacity errors, since different elements have somewhat different opacities. Rogers \& Iglesias (1998) estimate that such abundance uncertainties correspond to opacity uncertainties of order $5 \%$ at temperatures where ionization effects of the relevant elements yield a large contribution to the opacity (e.g., near $2 \times 10^{6} \mathrm{~K}$ for oxygen or neon). Turcotte et al. (1998) found that taking into account the opacity effects due to changes in the relative abundances of all the individual elements in $Z$ led to opacities that differed by up to $2 \%$ from the opacities tabulated for the standard scaled-solar metallicity, yielding sound speed differences of up to 0.001 and density differences up to 0.005 .

We did not attempt detailed element-by-element variations of the OPAL opacities in our models; nor did we test the effect of opacity variations in limited density or temperature ranges. Instead, we obtained a rough estimate of the maximum possible effects of uncertainties in heavy-element opacities by making an overall shift in the metallicity value $Z_{\kappa}$ used for interpolation in the OPAL opacity tables. Figure 2 illustrates the cases $Z_{\kappa}=0.9 Z$ ("low- $\kappa$ ") and $Z_{\kappa}=1.1 Z$ ("high- $\kappa$ "). This shift in the value of $Z_{\kappa}$ corresponds to an average shift in the opacities of order $10 \%$ over the solar interior $\left(2-5 \%\right.$ for $r \lesssim 0.4 R_{\odot}, \sim 10 \%$ for $0.4 R_{\odot}<r<0.7 R_{\odot}, \sim 15 \%$ for $0.7 R_{\odot}<r<0.92 R_{\odot}$, and $\sim 5 \%$ for $r>0.92 R_{\odot}$ ). As may be seen from Figure 2, such an opacity shift of order $10 \%$ yields changes of up to 0.003 in $\Delta c / c$; the rms differences are 0.0016 in $\Delta c / c$ and 0.014 in $\Delta \rho / \rho$.

We tested cases where new OPAL opacities had been calculated for mixes in which the 


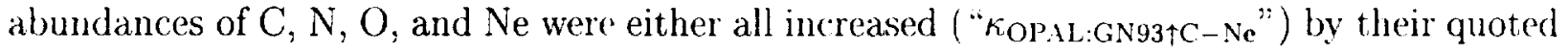

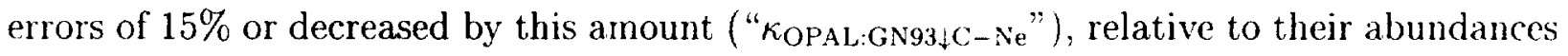
quoted by Grevesse \& Sauval (1998). Figure 2 illustrates an effect of up to 0.001 in the sound speed from such a change. However, we used a value of $Z / X=0.0245$ for all of the models in Figure 2, which is not strictly consistent with such large abundance changes: since $\mathrm{C}, \mathrm{N}, \mathrm{O}$, and Ne comprise the major portion of $Z$, a $15 \%$ change in their abundances should correspond to a change of $\sim 12 \%$ in $Z / X$ as well. We discuss such a self-consistent composition-plus- $Z / X$ variation under $Z / X$ effects below.

Solar abundance $(\mathrm{Z} / \mathrm{X})$ effects: Our reference standard solar model used the observational value of $Z / X=0.0245$ from Grevesse \& Noels (1993), since their mixture was the one for which the standard OPAL opacity tables ("$\kappa_{\text {OPAL:GN93") were available. The }}$ close-dashed curve in Figure 3 demonstrates the effects of using the Grevesse \& Noels (1993)

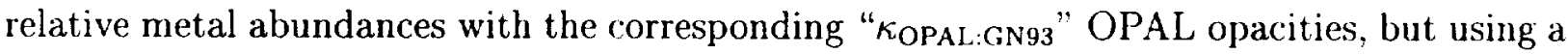
$13 \%$ higher value of $Z / X=0.0277$, the older value that had been recommended by Grevesse. (1984); the maximum sound speed difference relative to the reference standard solar model is 0.0030 , and the rms differences are 0.0018 in $\Delta c / c$ and 0.017 in $\Delta \rho / \rho$. The dotted curve in Figure 3 illustrates a similar case with a $6 \%$ lower value of $Z / X=0.023$, as recommended by the more recent work of Grevesse \& Sauval (1998); the maximum sound speed difference is 0.0016 , with rms differences of 0.0010 in $\Delta c / c$ and 0.009 in $\Delta \rho / \rho$. (Note that most of the above effect comes from the different opacity that results from the changed solar $Z$ value, as may be seen by comparing with the "high- $\kappa$ " and "low- $\kappa$ " curves in Fig. 2.) However, these comparisons are not strictly self-consistent, since it is the changes in the individual elemental abundances of the metals that adds up to yield the changed $Z / X$ ratio. Using the old abundance pattern of Grevesse (1984) and newly-computed OPAL opacities appropriate

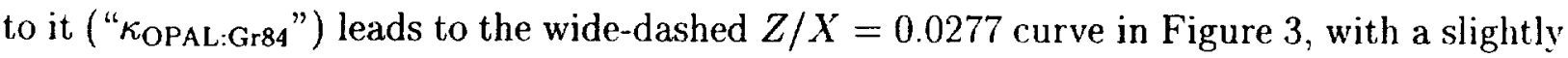
smaller maximum sound speed difference (of 0.0025) and rms differences (of 0.0014 in $\Delta c / c$ and 0.011 in $\Delta \rho / \rho$ ). Similarly, using the Grevesse \& Sauval (1998) abundance pattern and

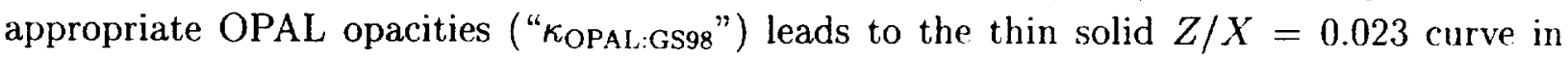
Figure 3, reducing the maximum sound speed difference to 0.0012 and the rms differences to 0.0006 in $\Delta c / c$ and 0.004 in $\Delta \rho / \rho$.

Strictly, the uncertainty resulting from observational solar abundance errors can be estimated by varying the solar abundance values of Grevesse \& Sauval (1998) within their quoted uncertainties, obtaining OPAL opacities with these revised compositions, calculating the resulting $Z / X$ values, and running solar models with these self-consistent sets of input values. We have done this for two cases. Rather than performing large numbers of random variations of the abundances, we tested a case which should give something close to the 
maximum effect. The elements $\mathrm{C}, \mathrm{N}, \mathrm{O}$, and Ne not only comprise the major part of the metallicity but also have relatively large errors of $\sim 15 \%$, and unlike other elements with large errors one cannot get a "better" value by using the meteoritic abundance instead. We therefore considered cases where $\mathrm{C}, \mathrm{N}, \mathrm{O}$, and Ne were either all increased by $15 \%(Z / X=$

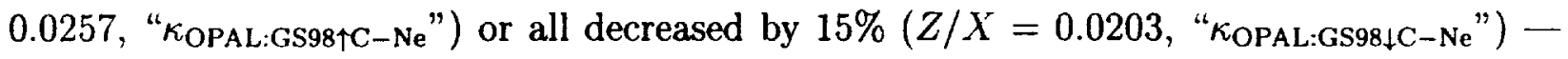
i.e., these self-consistent abundance variations correspond to $12 \%$ variations in $Z / X$. As illustrated in Figure 3, these cases lead to variations in the solar sound speed of up to 0.003

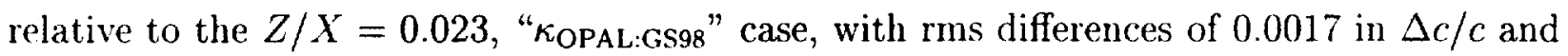
0.012 in $\Delta \rho / \rho$.

Morel et al. (1997) considered the effect of a $6 \%$ increase in $Z / X$, finding a maximum difference of 0.0007 in their sound speed and 0.003 in their density; this would imply a significantly lower sensitivity to $Z / X$ than we found. This is probably due to the fact that the models in which they tested $Z / X$ variations did not consider the effect on the opacities of the temporal and spatial variations in the heavy element abundances that arise from diffusion, but merely used opacities appropriate to a constant metallicity equal to the protosolar value $\left(Z_{\kappa}=Z_{0}\right.$, as in our "const- $Z_{\kappa}$ " case discussed above).

Nuclear rate effects: Figure 4 demonstrates that the uncertainty in the ${ }^{1} \mathrm{H}\left(p, \nu e^{+}\right)^{2} \mathrm{H}$ nuclear burning rate (the $p p$ rate) has a significant impact on the solar sound speed. Our reference standard solar model used the recommended nuclear rates from the NACRE compilation (Angulo et al. 1999). These authors also supply "high" and "low" cases to indicate the allowed uncertainty range of each nuclear rate; in the case of the $p p$ reaction, the high case is $8 \%$ above the recommended rate and the low case is $3 \%$ below it. We have tested the effects of nuclear rate uncertainties by computing variant standard solar models using high and low NACRE rate values. Figure 4 demonstrates that a high $p p$ rate is preferable, if all other parameters are kept constant: the high $p p$ rate gives good agreement with the helioseismic reference profiles, except in the Sun's central regions where the helioseismic observations are the poorest. Our models indicate that a change of $5 \%$ in the $p p$ rate yields changes of up to 0.003 in the sound speed, $(0.0014$ in the regions accurately probed by helioseismology, outside the core); the rms changes in such a case would be 0.0009 in $\Delta c / c$ and 0.018 in $\Delta \rho / \rho$. Antia \& Chitre (1999) also tested the effects of changes in the $p p$ rate on the helioseismic profiles, concluding that a relatively high $p p$ rate is preferred, consistent with our results discussed above.

Figure 4 also demonstrates that the uncertainty of $\pm 20 \%$ in the ${ }^{3} \mathrm{He}(\alpha, \gamma)^{7} \mathrm{Be}$ reaction leads to only a minor effect: a maximum change of 0.001 (or 0.0003 outside the core), with an rms relative change of 0.0002 in $\Delta c / c$ and 0.004 in $\Delta \rho / \rho$. Basu et al. (2000) considered the 


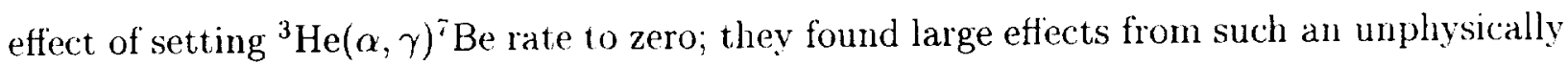
extreme change. Setting the rate to zero is equivalent to a $100 \%$ change, 5 times as large as the $20 \%$ considered by us; thus it is consistent that their published effect is about 5 times as large as ours.

We also tested the effects of the $\pm 6 \%$ uncertainty in the ${ }^{3} \mathrm{He}\left({ }^{3} \mathrm{He}, 2 p\right){ }^{4} \mathrm{He}$ reaction and of the $\pm 30 \%$ uncertainty in the ${ }^{14} \mathrm{~N}(p, \gamma){ }^{15} \mathrm{O}$ reaction (which dotermines the CNO-cycle rate). Such changes in these rates led to negligible effects on the sound speed and density profiles; we have not plotted these profiles in Figure 4, since they would be essentially superimposed on that of the reference standard solar model.

It is not surprising that the uncertainty in the $p p$ rate has the largest effect on the sound speed and density profiles, since it is the basic rate that determines the overall p-p chain burning rate.

We also computed a model using the previous standard set of nuclear rates, namely, the Kellogg nuclear rate compilation of Caughlan \& Fowler (1988). The resulting sound speed and density profiles are shown in Figure 4. With us still in Kellogg, carrying out this work in an office directly below his long-time office, it is especially gratifying for us to see that Willy Fowler's last published $p p$ rate yields such good agreement with the current helioseismic reference profiles (the largest differences being near the center, where the observations are least accurate).

Diffusion effects: There are uncertainties in the diffusion coefficients; Proffitt (1994) estimates a $15 \%$ uncertainty in the diffusion constant of helium relative to hydrogen, and a $\sim 50 \%$ uncertainty in the diffusion constant for oxygen relative to hydrogen. Figure 5 shows that an increase or decrease of $20 \%$ in the helium diffusion constants has only a modest effect: a maximum change of 0.001 in the sound speed, with rms changes of 0.0008 in $\Delta c / c$ and 0.007 in $\Delta \rho / \rho$. The effect of increasing or decreasing the heavy element diffusion constants by $40 \%$ has an even smaller effect: a maximum change of 0.0006 in the sound speed, with rms changes of 0.0004 in $\Delta c / c$ and 0.004 in $\Delta \rho / \rho$.

\subsection{Solar Convective Envelope Depth}

One of the key results of helioseismic observations is a highly precise value for the position $R_{c e}$ of the base of the solar surface convective region: Basu \& Antia (1997) report a value of $R_{c e}=0.713 \pm 0.001 R_{\odot}$. Our reference standard solar model is in agreement with this value, having $R_{c e}=0.7134 R_{\odot}$ (see Table 1), independent of whether we used the OPAL 
or the MHD equation of state at $\log \rho \lesssim-2$. The low and high $Z / X$ values are marginally inconsistent with the observed value, lying at $R_{c e}=0.7181$ and $0.7096 R_{\odot}$, respectively. The uncertainty of \pm 0.05 Gyr does not make a significant difference in $R_{c e}$ (see Table 1 ). As far as the uncertainties in the nuclear reaction rates are concerned, only the $p p$ rate has a significant effect on $R_{c e}$, of $\pm 0.002 R_{\odot}$. Uncertainties in molecular opacities do not have a significant effect on $R_{c e}$, but changing from the old 1985 LAOL opacities to the 1995 OPAL opacities does yield a large improvement (of $0.006 R_{\odot}$ ); the remaining uncertainties in the 1995 OPAL opacities might thus be expected to have a small but possibly significant effect on $R_{c e}$. The uncertainty in the diffusion constants for helium does have a significant influence $\left( \pm 0.003 R_{\odot}\right)$, but uncertainties in the diffusion constants for the heavy elements do not (effects $\lesssim 0.001 R_{\odot}$ ).

Note that the cases favored by the sound speed profiles at the one- to two-sigma significance level (high opacities, high $Z / X$, or high $p p$ rate) are disfavored by the observed $R_{c e}$ value at about the same significance level.

\subsection{Solar Helium Abundance}

Another key result of helioseismic observations is a precise value of solar envelope helium mass fraction $Y_{e}$ (this value is lower than the Sun's initial helium abundance, due to diffusion). Inferring the solar helium abundance requires use of a (theoretical) equation of state as well as the helioseismic frequency observations that probe the solar convective region, particularly the He II ionization zone at $0.975 R_{\odot} \lesssim r \lesssim 0.985 R_{\odot}$ (Richard et al. 1998). In recent work, there has been excellent agreement on the value of $Y_{e}$ when the OPAL equation of state was used: $Y_{e}=0.249 \pm 0.001$ (Basu \& Antia 1995), $Y_{e}=0.248 \pm 0.006$ (Kosovichev 1997), $Y_{e}=0.248 \pm 0.001$ (Basu 1998), and $Y_{e}=0.248 \pm 0.002$ (Richard et al. 1998). A larger scatter has resulted when the MHD equation of state was used: $Y_{e}=0.242 \pm 0.003$ (Pérez Hernández \& Christensen-Dalsgaard 1994), $Y_{e}=0.246 \pm 0.001$ (Basu \& Antia 1995), $Y_{\epsilon}=0.232 \pm 0.006$ (Kosovichev 1997), $Y_{e} \approx 0.252$ (Basu 1998), and $Y_{e} \approx 0.242$ (Richard et al. 1998). The OPAL equation of state is expected to be more accurate than the MHD equation of state over the bulk of the convective envelope, since it was designed for use at $\log \rho \lesssim-2$ (which occurs in the Sun at $r \gtrsim 0.942 R_{\odot}$ ); however, the He II ionization zone occurs in a region the MHD equation of state was specifically designed for, and where it may actually be more accurate than the OPAL equation of state (see, e.g., Richard et al. 1998). In summary, the helioseismic observations appear to constrain the solar envelope helium abundance to the range $0.24 \lesssim Y_{e} \lesssim 0.25$.

Our theoretical reference standard solar model is in excellent agreement with this, having 
$Y_{e}=0.2424$ independent of whether we used the OPAL or the MHD equation of state at $\log \rho \lesssim-2$. Low and high $Z / X$ values yield $Y_{e}$ values of 0.2344 and 0.2517 , respectively, on the borderline of acceptability. Uncertainties in the solar age, the nuclear rates, and opacities have only a negligible effect on $Y_{e}$; uncertainties in the diffusion constants have a minor effect, but still yield acceptable values (see Table 1).

\subsection{Solar Lithium Abundance}

The present observed solar surface lithium abundance is $\log \varepsilon\left({ }^{7} \mathrm{Li}\right)=1.10 \pm 0.10$ as compared to the initial value of $\log \varepsilon\left({ }^{7} \mathrm{Li}\right)=3.31 \pm 0.04$ obtained from meteorites (Grevesse \& Sauval 1998), where $\log \varepsilon\left({ }^{7} \mathrm{Li}\right)=\log \left(N_{\mathrm{Li}} / N_{\mathrm{H}}\right)+12$ for number densities $N_{\mathrm{Li}}$ and $N_{\mathrm{H}}$ of lithium and hydrogen, respectively. The solar surface lithium depletion factor $f_{\mathrm{Li}}$, relative to its initial value, is thus observed to be $f_{\mathrm{Li}}=160 \pm 40$. Solar surface lithium can be depleted due to three causes: (1) lithium burning during the pre-main-sequence evolution, when the surface convection still reaches deply into the interior: (2) rotationally induced mixing on the main sequence, which transports lithium down from the convective envelope to regions hot enough for lithium burning; (3) mass loss on the main sequence, which can cause the convective envelope to move inwards and engulf lithium-depleted regions. In this paper, we only consider the first of these, namely, the pre-main-sequence lithium destruction; rotational mixing is beyond the scope of this paper, and main sequence mass loss is discussed in the companion "Our Sun V" paper (Sarkmann \& Boothroyd 2001). Our reference standard solar model had a pre-main-sequence lithium depletion factor $f_{\mathrm{Li}}=24$, as shown in Table 1.

Pre-main-sequence lithium depletion depends quite sensitively on the structure of the solar models during that stage of evolution. We give our results in terms of the solar lithium depletion factor $f_{\mathrm{Li}}$, which is shown in Table 1 . The zoning did not affect the lithium depletion factor significantly; nor did the uncertainties in the solar age, luminosity, and radius. Changes in the equation of state in the outermost regions can have a small effect $(\lesssim 30 \%)$ on the lithium depletion factor: the "OPALeos-mid'T" and "OPALeos-low'T" cases had lithium depletion factors of $f_{\mathrm{Li}}=17$ and 19 , respectively. as opposed to $f_{\mathrm{Li}}=24$ for the standard "OPALeos-hiT" case.

The pre-main-sequence lithium depletion is extremely sensitive to both low-temperature. and high-temperature opacities; use of the Sharp (1992) molecular opacities instead of the Alexander \& Ferguson (1994) ones halved the lithium depletion factor $\left(f_{\mathrm{Li}}=10\right)$, as did our "low- $\kappa$ " test case $\left(f_{\mathrm{Li}}=10\right)$, while our "high- $\kappa$ " test case nearly tripled it $\left(f_{\mathrm{Li}}=71\right)$. There is also a relatively large sensitivity to the uncertainty in the observed solar abundances. A

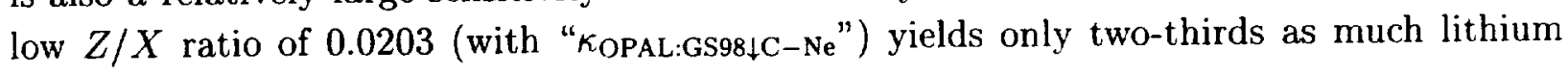


depletion $\left(f_{\mathrm{li}}=15\right.$ ), while a high $Z / X$ ratio of 0.0257 (with "hopal:Gsuds:-Ne") yields half again as much lithium depletion $\left(f_{\mathrm{Li}}=35\right)$.

Uncertainties in the diffusion constants can affect the lithium depletion factor significantly, primarily due to the effect of the different initial composition; our diffusion test cases have depletion factors ranging from $f_{\mathrm{Li}}=18$ to 33 .

Except for the ${ }^{7} \mathrm{Li}+p$ rate, uncertainties in the nuclear rates have almost no effect on the extent of lithium depletion. For the ${ }^{7} \mathrm{Li}+p$ rate, the $\pm 14 \%$ uncertainty quoted by the NACRE compilation (Angulo et al. 1999) corresponds to an uncertainty of about $\pm 50 \%$ in the depletion factor (i.e., a range in the from $f_{\mathrm{Li}}=16$ to 38 ).

Solar beryllium abundance: The observed solar beryllium abundance is $\log \varepsilon\left({ }^{9} \mathrm{Be}\right)=$ $1.40 \pm 0.09$, consistent with no depletion relative to the meteoritic value of $\log \varepsilon\left({ }^{9} \mathrm{Be}\right)=$ $1.42 \pm 0.04$. These values imply that solar beryllium cannot have been depleted by more than a factor of 2 (3- $\sigma$ upper limit). Our solar models all had negligible amounts of beryllium depletion, of order $1 \%$.

\subsection{Solar neutrinos}

We will not devote much space to the predicted solar neutrino values, since it has long been concluded that matching the observed neutrino capture rates requires not revised astrophysics but new neutrino physics, e.g., Mikheyev-Smirnov-Wolfenstein (MSW) neutrino oscillation effects (see, e.g., Bahcall, Basu, \& Pinsonneault 1998a; Suzuki 1998; Basu et al. 2000; Bahcall et al. 2001). In Table 1 we present the theoretically predicted neutrino capture rates for the ${ }^{37} \mathrm{Cl}$ and ${ }^{71} \mathrm{Ga}$ experiments, and the predicted flux of ${ }^{8} \mathrm{~B}$ neutrinos. As is normally obtained, our theoretical predicted neutrino rates are much in excess of the observed values, i.e., 6.4 to $8.9 \mathrm{SNU}$ is predicted for the ${ }^{37} \mathrm{Cl}$ experiment, as compared to the observed value of $2.56 \pm 0.23$ SNU (Davis 1994; Cleveland et al. 1998; Lande 2001), and 127 to $141 \mathrm{SNU}$ for the ${ }^{71} \mathrm{Ga}$ experiments, as compared to the observed value of $74.5 \pm 5.7 \mathrm{SNU}$ (combined value from SAGE and GALLEX+GNO: Hampel et al. 1999; Abdurashitov et al. 1999; Altmann et al. 1999; Gavrin 2001; Ferrari 2001). Likewise, the models predict ${ }^{8} \mathrm{~B}$ fluxes of 4.4 to $6.3 \times 10^{6} \mathrm{~cm}^{-2} \mathrm{~s}^{-1}$, as compared to the value of $(2.32 \pm 0.08) \times 10^{6} \mathrm{~cm}^{-2} \mathrm{~s}^{-1}$ measured by Super-Kamiokande (Fukuda et al. 2001).

We wish to thank Prof. Charles A. Barnes and Prof. Yuk L. Yung for thoughtful discussions and encouragement. We are grateful to Prof. Robert D. McKeown for the support 
provided by the W. K. Kellogg Radiation Laboratory. One of us (I.-J. S.) wishes to thank Alexandra R. Christy, her daughter, and Prof. Robert F. Clristy, her husband, for their supportiveness, and Robert F. Christy for critical analysis and helpful comments. One of us (A. I. B.) wishes to thank Prof. Peter G. Martin and Prof. J. Richard Bond for their support, and M. Elaine Boothroyd, his wife, for her patience and encouragement. This work was supported by a grant NAG5-7166 from the Sun-Earth Connection Program of the Supporting Research and Technology and Suborbital Program in Solar Physics of the National Aeronautics and Space Administration, and by the National Science Foundation grant NSF-0071856 to the Kellogg Radiation Laboratory. 


\section{REFERENCES}

Abdurashitov, J.N. et al. 1999, Phys. Rev. Lett., 83, 4686 (GALLEX Collaboration)

Alexander, D. R., \& Ferguson, J. W. 1994, ApJ, 437, 879

Altmann, M., et al. 1999, Phys. Lett. B, 490, 16 (GNO Collaboration)

Anders, E., \& Grevesse, N. 1989, Geochim. Cosmochim. Acta, 53, 197

Angulo, C. et al. 1999, Nucl. Phys. A, 656, 3

Antia, H. M. 1998, A\&A, 330, 336

Antia, H. M., \& Chitre, S. M. 1999, A\&A, 347, 1000

Aufderheide, M. B., Bloom, S. B., Resler, D. A., \& Goodman, C. D. 1994, Phys. Rev. C, 49, 678

Bahcall, J. N., Bahcall, N. A., \& Shaviv, G. 1968, Phys. Rev. Lett., 20, 1209

Bahcall, J. N., Basu, S., \& Pinsonneault, M.H. 1998, Phys. Lett. B, 433, 1

Bahcall, J. N., Chen, X. L., \& Kamionkowski, M. 1998, Phys. Rev. C, 57, 2756

Bahcall, J. N., \& Moeller, C. P. 1969, ApJ, 155, 511

Bahcall, J. N., Pinsonneault, M. H., \& Basu, S. 2001, ApJ, 555, 990

Bahcall, J. N., Pinsonneault, M. H., \& Wasserburg, G. J. 1995, Rev. Mod. Phys., 67, 781

Bahcall, J. N., \& Ulrich, R. K. 1988, Rev. Mod. Phys., 60, 297

Basu, S. 1998, MNRAS, 298, 719

Basu, S., \& Antia H. M. 1995, MNRAS, 276, 1402

Basu, S., \& Antia H. M. 1997, MNRAS, 287, 189

Basu, S., Pinsonneault, M. H., \& Bahcall, J. N. 2000, ApJ, 529, 1084

Boothroyd, A. I., \& Sackmann, I.-J. 1999, ApJ, 510, 232

Brown, T. M., \& Christensen-Dalsgaard, J. 1998, ApJ, 500, L195

Brun, A. S., Turck-Chièze, S., \& Zahn, J. P. 1999, ApJ, 525, 1032 
Caughlan, G. R., \& Fowler, W. A. 1988. Atomic Data Nucl. 1)ata Tables, 40, 283

Chaplin, W. J., Elsworth, Y., Howe, R., Isaak, G. R., McLeod, C. P.. Miller, B. A., van der Raay, H. B., Wheeler, S. J., \& New, R. 1996, Sol. Phys., 168, 1

Christensen-Dalsgaard, J., \& Däppen, W. 1992, A\&A Rev., 4, 267

Cleveland, B. T., Daily, T., Davis, R., Jr., Distel, J. R., Lande, K.. Lee, C. K., Wildenhain, P. S., \& Ullman, J. 1998, ApJ, 496, 505

Cohen, E. R., \& Taylor, B. N. 1986, Codata Bulletin No. 63 (New York: Pergamon)

Crommelynck, D., Fichot, A., Domingo, V., \& Lee, R. 1996, Geophys. Res. Lett., 23, 2293

Däppen, W., Mihalas, D., Hummer, D. G., \& Mihalas, B. 1988, ApJ, 332, 261

Davis, R. 1994, Prog. Part. Nucl. Phys., 32, 13

Elliot, J. R., \& Kosovichev, A. G. 1998, Ap.J, 500, L199

Ferrari, N. 2001, Nucl. Phys. B, 100, 48

Fröhlich, C., \& Lean, J. 1998, Geophys. Res. Lett., 25, 4377

Fukuda,S., et al. 2001, Phys. Rev. Lett., 86, 5651 (Super-Kamiokande Collaboration)

Garrin, V. N. 2001, Nucl. Phys. B, 91. 36

Geiss, J. 1973, in Proc. 13th Intl. Cosmic Ray Conf., vol. 5 (Denver: Cniv. of Denver), 3375

Geiss, J., \& Bochsler, P. 1991, in The Sun in Time, ed. C. Sonnett, M. Giampapa, \& M. Matthews (Tucson: Univ. Arizona Press), 98

Graboske, H. C., DeWitt, H. E., Grossman, A. S., \& Cooper, M. S. 1973, ApJ, 181, 457

Grevesse, N. 1984, Phys. Scr, T8, 49

Grevesse, N., \& Noels, A. 1993, in Origin and Evolution of the Elements, ed. N. Prantzos, E. Vangioni-Flam, \& M. Cassé (Cambridge: Cambridge University Press), 15

Grevesse, N., \& Sauval, A. J. 1998, Space Sci. Rev., 85, 161

Gruzinov, A. V., \& Bahcall, J. N. 1997, ApJ, 490, 437

Gruzinov, A. V., \& Bahcall, J. N. 1998, ApJ, 504, 996 
Guenther. D. B., Demarque, P., Kim, Y.-C., \& Pinsonneault, M. H. 1992, ApJ, 387.372

Guzik, J. A., \& Swenson, F. J. 1997, ApJ, 491, 967

Hampel, W., et al. 1999, Phys. Lett. B, 447, 127 (GALLEX Collaboration)

Iglesias, C. A., \& Rogers, F. J. 1996, ApJ, 464, 943

Itoh, N., Totsuji, H., Ichimaru, S., \& DeWitt, H. E. 1979, ApJ, 234, 1079; erratum 1979 , ApJ, 239, 415

Ichimaru, S., \& Utsumi, K. 1983, ApJ, 269, L51

Kerridge, J. F., Signer, P., Wieler, R., Becker, R. H., \& Pepin, R. O. 1991, in The Sun in Time, ed. C. Sonnett, M. Giampapa, \& M. Matthews (Tucson: Univ. Arizona Press), 389

Kosovichev. A. G. 1997, in AIP Conf. Proc. 385, Robotic Exploration Close to the Sun: Scientific Basis, ed. S. R. Habbal (Woodbury, NY: Amer. Inst. Phys.), 159

Lande, K. 2001, in Proc. of the 2000 XIX Conf. on Neutrino Physics \& Astrophysics (Amsterdam: Elsevier), in press

Lean, J. 2000, Geophys. Res. Lett., 27, 2425

Morel, P., Provost, J., \& Berthomieu, G. 1997, Adt, 327, 349

Pérez Hernández, F., \& Christensen-Dalsgaard, J. 1994, MNRAS, 269, 475

Proffitt, C. R. 1994, ApJ, 425, 849

Rhodes, E. J., Jr., Kosovichev, A. G., Schou, J., Scherrer, P. H., \& Reiter, J. 1997, Sol. Phys., 175,287

Richard, O., Dziembowski, W. A., Sienkiewicz, R., \& Goode, P. R. 1998, A\&A, 338, 756

Richard, O., Vauclair, S., Charbonnel, C., \& Dziembowski, W. A. 1996, ApJ, 312, 1000

Rogers, F. J., \& Iglesias, C. A. 1992, ApJS, 79, 507

Rogers, F. J., \& Iglesias, C. A. 1992, Space Sci. Rev., 85, 61

Rogers, F. J., Swenson, F. J., \& Iglesias, C. A. 1996, ApJ, 456, 902

Sackmann, I.-J., \& Boothroyd, A. I. 2001, ApJ, submitted (Our Sun V) 
Sackmann, I.-J., Boothroyd. A. 1., \& Kraemer. K. E. 1993, Ap.J, 418.457

Salpeter, E. E. 1955, ApJ, 121, 161

Sharp, C. M. 1992, A\&AS, 94, 1

Suzuki, Y. 1998, Space Sci. Rev., 85, 91

Thoul, A. A., Bahcall, J. N., \& Loel,, A. 1994, ApJ, 421, 828

Tomczyk, S., Streander, K., Card, Ci.. Elmore, D., Hull, H., \& Caccani, A. 1995, Sol. Phys., Sol. Phys., 159, 1

Turcotte, S., \& Christensen-Dalsgaard, J. 1998, Space Sci. Rev. 85, 133

Turcotte, S., Richer, J., Michand, G., Iglesias, C. A., \& Rogers, F. J. 1998, ApJ, 504, 539

Ulrich, R. K., \& Rhodes, E. R., Jr. 1983. Ap.J, 265, 551 

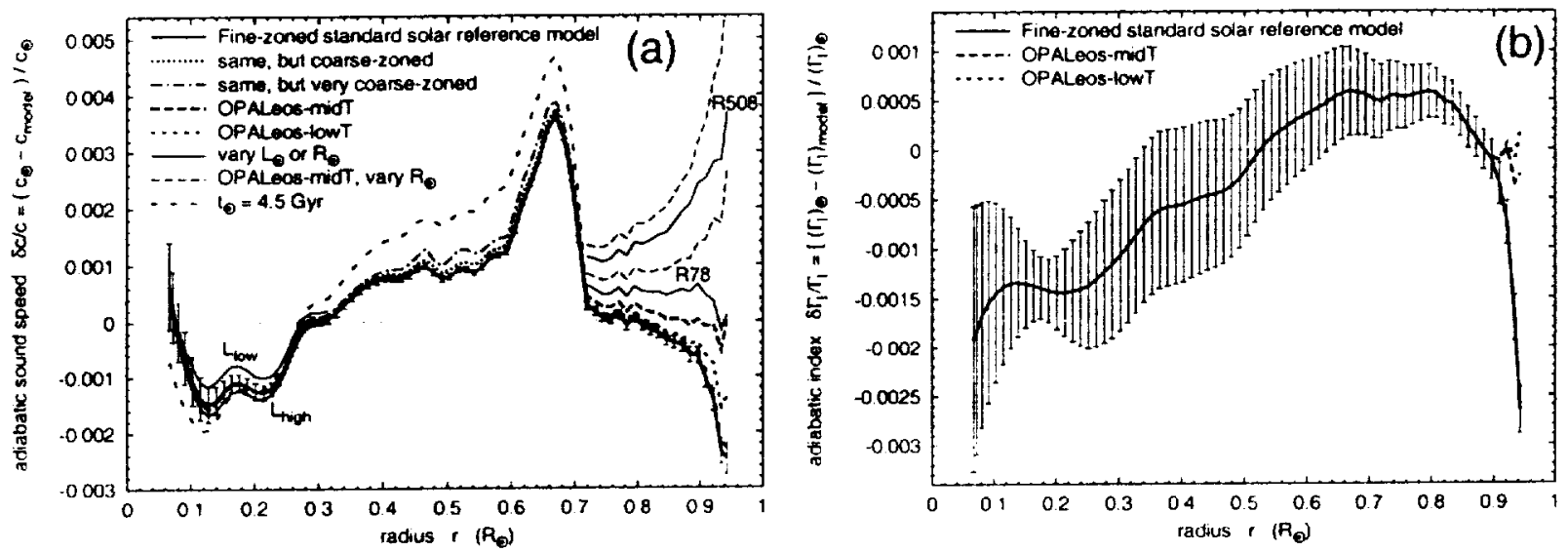

Fig. 1.- Changing the zoning, the outer-envelope equation of state, or the solar radius, luminosity, or age: the effects on (a) the adiabatic sound speed $c$, and (b) the adiabatic in$\operatorname{dex} \Gamma_{1}$. The fine-zoned reference standard solar model (solid line - errorbars give statistical error in the inferred helioseismic profile) switches from the OPAL to the MHD equation of state as the density drops below $\log \rho=-2$ (i.e., $r \approx 0.94 R_{\odot}$ ); the coarse zoned (dotted line) and very coarse zoned (dot-dashed line) models are the same except for the zoning. The fine-zoned "OPALeos-midT" (short-dashed line) and "OPALeos-lowT" (dashed line) models have the equation-of-state switchover at $\log T=4.0$ and $\log T=3.75$, respectively. The wide-dashed line shows the effect of using a reduced solar age (actually, $\sim 10-\sigma$ below the lower limit of $4.55 \mathrm{Gyr}$ ). For clarity, luminosity and radius effects are shown only in regions where they have a visible effect. For $r<0.3 R_{\odot}$, thin solid lines show the effects of using the maximum (" $L_{\text {high }}$ ") and minimum (" $L_{\text {low" }}$ ) values of $L_{\odot \odot}$. For $r \geq 0.71 R_{\odot}$, thin solid lines show the effects of using $R_{\odot}=695.78 \mathrm{Mm}$ ("R78") or $R_{\odot}=695.508 \mathrm{Mm}$ ("R508"), while thin dashed lines show the same radius effects for the "OPALeos-midT" case. 


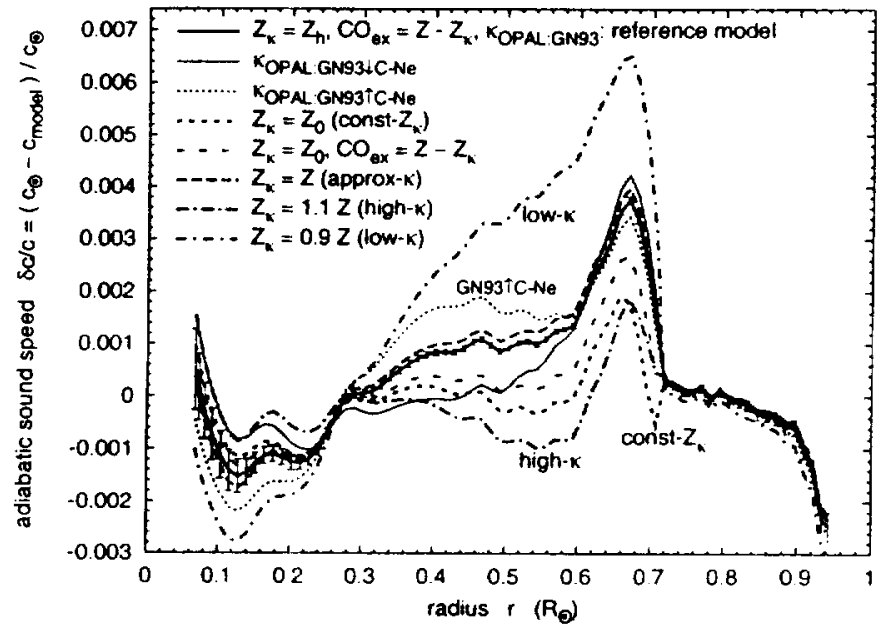

Fig. 2.- Effect on the sound speed of opacity uncertainties. The dot-dashed lines ("low$\kappa$ " and "high- $\kappa$ ") show the effect of a $\sim 10 \%$ overall change in the opacities relative to the reference standard solar model (thick solid line). Ignoring opacity effects due to relative CNO abundance changes from CNO-burning has little effect (dashed line), but ignoring opacity effects due to metallicity variations from diffusion leads to large errors (short-dashed and wide-dashed lines). Opacity uncertainties from uncertainties in relative metal abundances can be significant, even when the solar surface $Z / X$ ratio is unclianged (thin solid and dotted lines). 


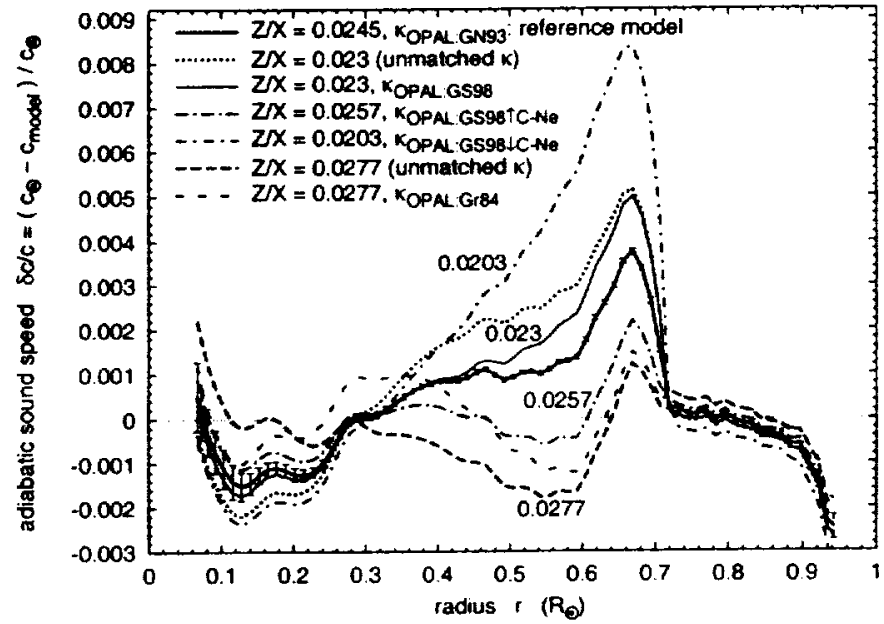

Fig. 3.-- Effect on the sound speed of uncertainties in the observed solar surface composition. Relative to the reference standard solar model at $Z / X=0.0245$ (thick solid line), switching to the more recent $Z / X=0.023$ value of Grevesse \& Sauval (1998) without including the corresponding changes in relative metal abundances (dotted line) has a larger effect than the

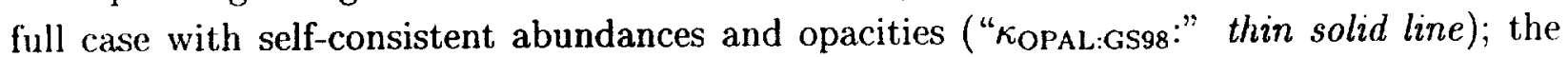
same is true when considering the older $Z / X=0.0277$ cases of Grevesse (1984) (dashed line

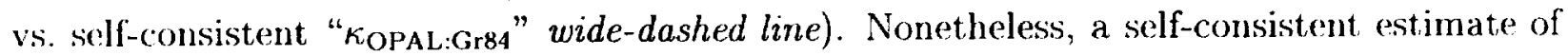
the uncertainties in the Grevesse \& Sauval (1998) composition (dot-dashed lines) shows a large effect (compare to the thin solid line). 


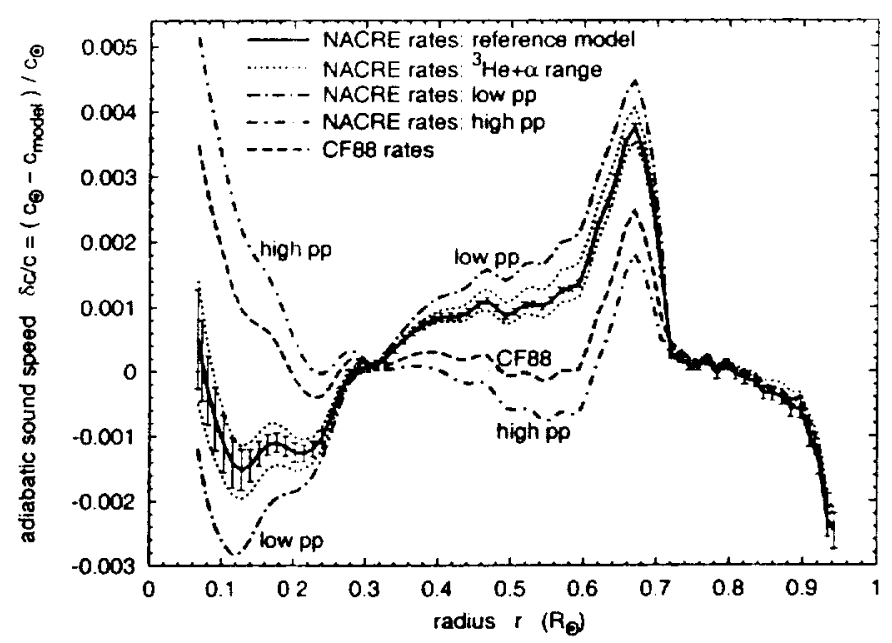

Fig. 4. - Effect on the sound speed of uncertainties in nuclear rates. The $\sim 5 \%$ uncertainty in the basic $p$ ) rate (dot-doshed lines) has a much larger effect than the $20 \%$ uncrertainty in the ${ }^{3} \mathrm{He}+\alpha$ rate (dotted lines); other rate uncertainties have negligible effects, and are not plotted. The difference between the reference standard solar model using the NACRE rates (solid line) and the model using the Caughlan \& Fowler (1988) rates ("CF88:" dashed line) is largely due to the higher $p p$ rate adopted by the latter authors.

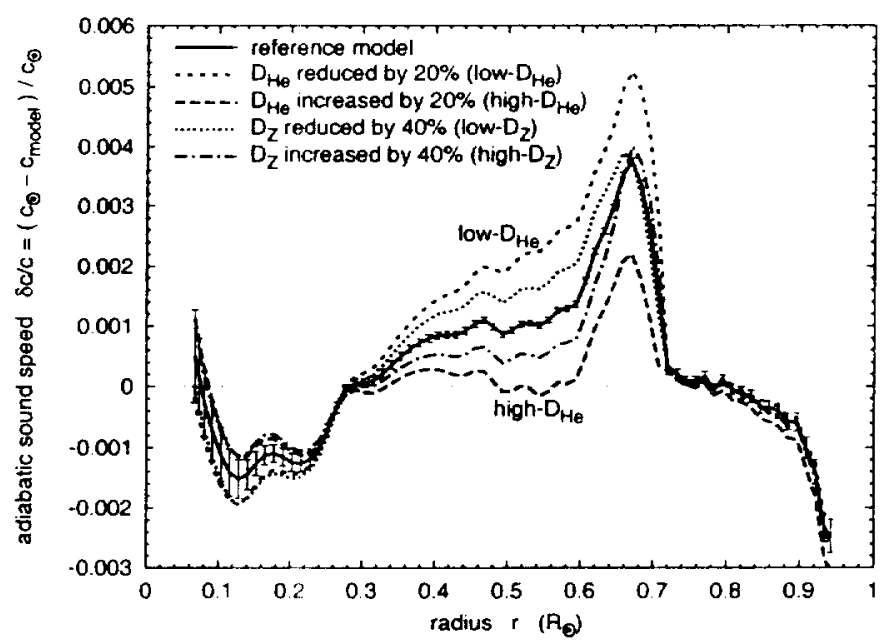

Fig. 5.- Effect on the sound speed of uncertainties in diffusion constants. A $20 \%$ variation in the diffusion constant for helium (short-dashed and long-dashed lines) has a larger effect than a $40 \%$ variation in the heavy element diffusion constants (dotted and dot-dashed lines) - the reference standard solar model is shown by the solid line. 
$-34-$

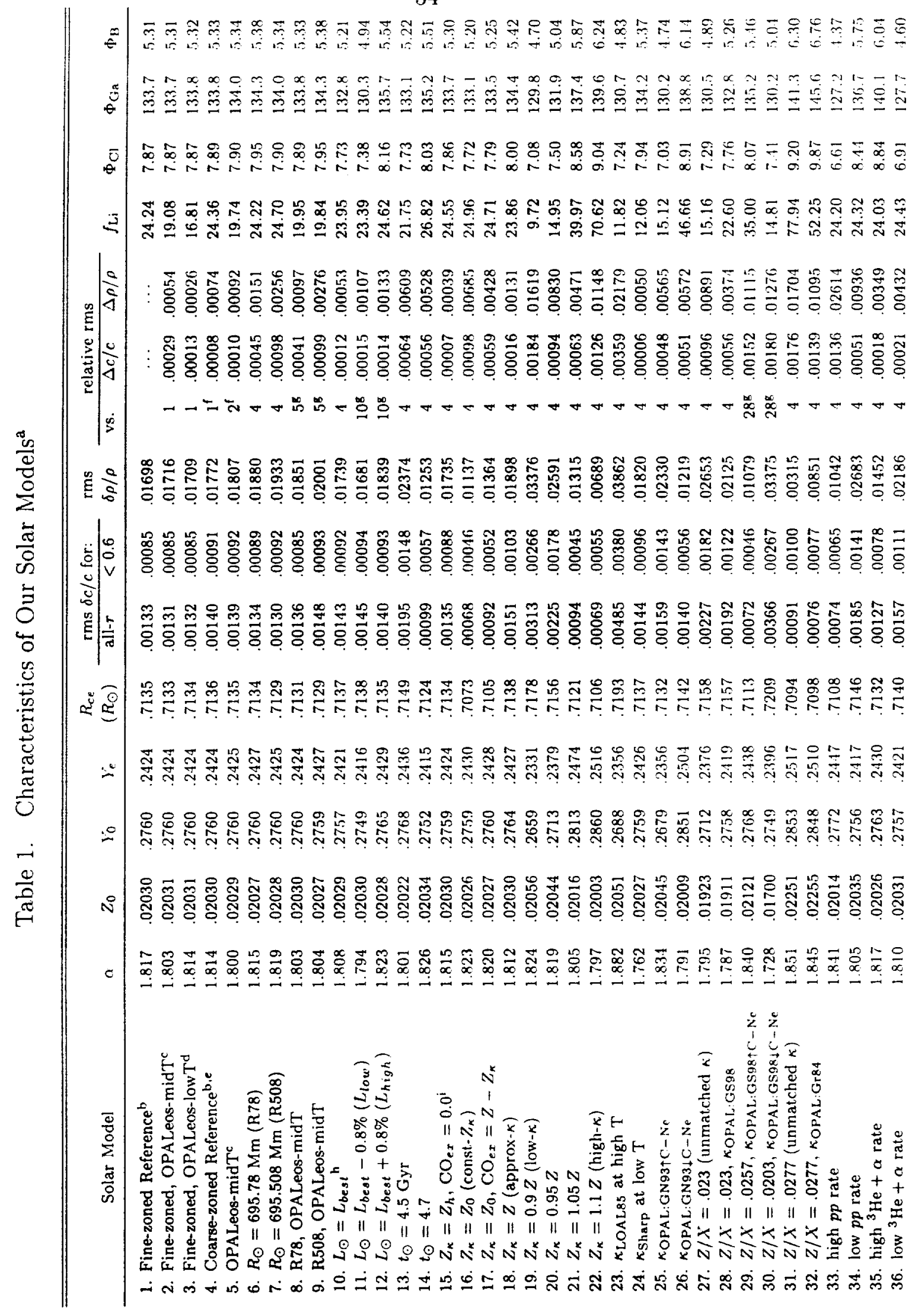




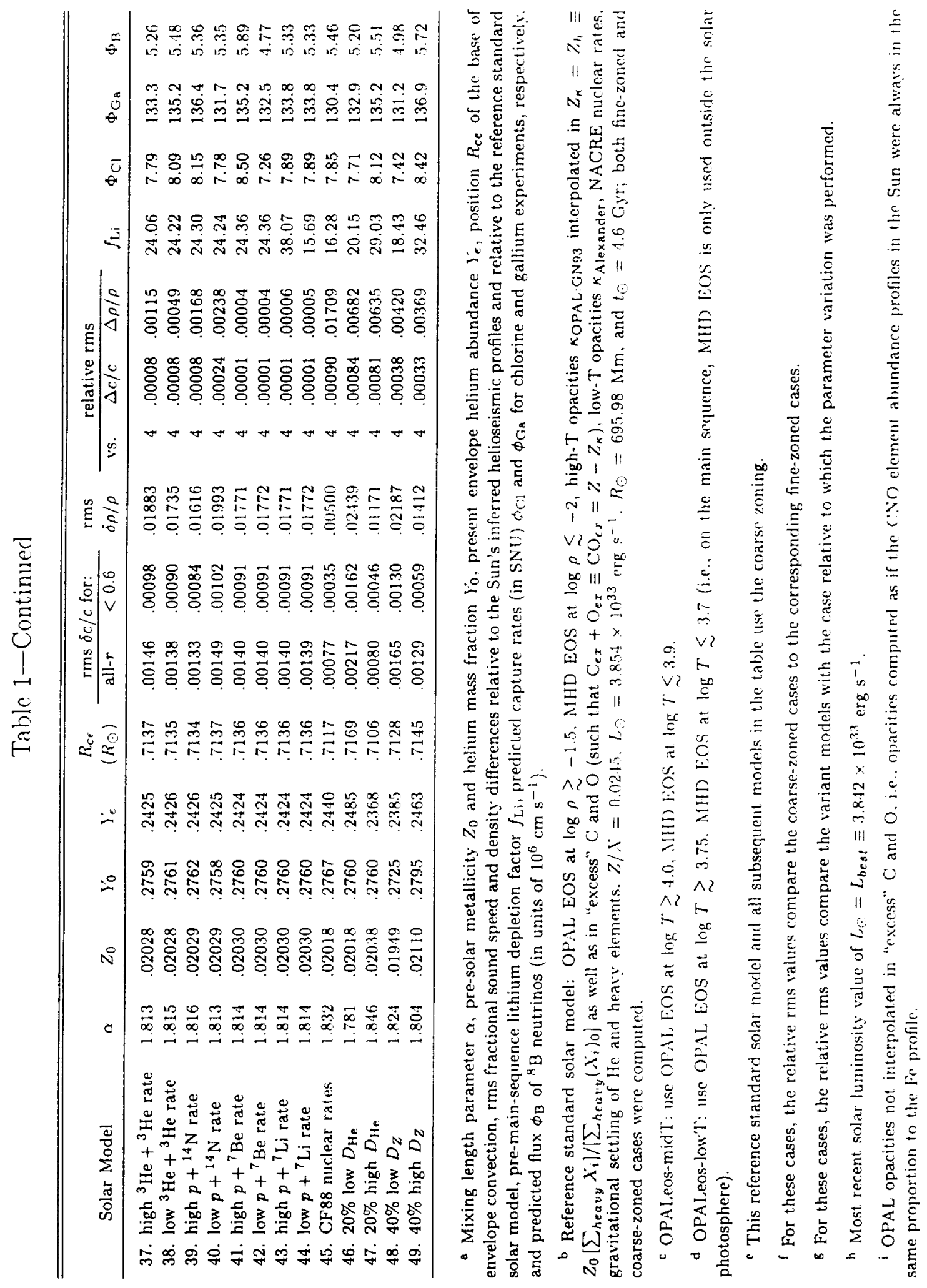


